\title{
A high-order positivity-preserving single-stage single-step method for the ideal magnetohydrodynamic equations
}

\author{
Andrew J. Christlieb ${ }^{\mathrm{a}}$, Xiao Feng ${ }^{\mathrm{b}, 1}$, David C. Seal ${ }^{\mathrm{c}}$, Qi Tang ${ }^{\mathrm{d}}$ \\ ${ }^{a}$ Department of Computational Mathematics, Science, and Engineering, Department of Mathematics, and \\ Department of Electrical and Computer Engineering, Michigan State University, East Lansing, MI 48824 USA \\ ${ }^{b}$ Department of Mathematics, Michigan State University, East Lansing, MI 48824, USA \\ ${ }^{c}$ Department of Mathematics, U.S. Naval Academy, 121 Blake Road, Annapolis, MD 21402, USA \\ ${ }^{d}$ Department of Mathematical Sciences, Rensselaer Polytechnic Institute, Troy, NY 12180, USA
}

\begin{abstract}
We propose a high-order finite difference weighted ENO (WENO) method for the ideal magnetohydrodynamics (MHD) equations. The proposed method is single-stage (i.e., it has no internal stages to store), single-step (i.e., it has no time history that needs to be stored), maintains a discrete divergence-free condition on the magnetic field, and has the capacity to preserve the positivity of the density and pressure. To accomplish this, we use a Taylor discretization of the Picard integral formulation (PIF) of the finite difference WENO method proposed in [SINUM, 53 (2015), pp. 18331856], where the focus is on a high-order discretization of the fluxes (as opposed to the conserved variables). We use the version where fluxes are expanded to third-order accuracy in time, and for the fluid variables space is discretized using the classical fifth-order finite difference WENO discretization. We use constrained transport in order to obtain divergence-free magnetic fields, which means that we simultaneously evolve the magnetohydrodynamic (that has an evolution equation for the magnetic field) and magnetic potential equations alongside each other, and set the magnetic field to be the (discrete) curl of the magnetic potential after each time step. In this work, we compute these derivatives to fourth-order accuracy. In order to retain a single-stage, single-step method, we develop a novel Lax-Wendroff discretization for the evolution of the magnetic potential, where we start with technology used for Hamilton-Jacobi equations in order to construct a non-oscillatory magnetic field. The end result is an algorithm that is similar to our previous work [JCP, 268, (2014), pp. 302-325], but this time the time stepping is replaced through a Taylor method with the addition of a positivitypreserving limiter. Finally, positivity preservation is realized by introducing a parameterized flux limiter that considers a linear combination of high and low-order numerical fluxes. The choice of the free parameter is then given in such a way that the fluxes are limited towards the low-order solver until positivity is attained. Given the lack of additional degrees of freedom in the system, this positivity limiter lacks energy conservation where the limiter turns on. However, this ingredient can be dropped for problems where the pressure does not become negative. We present two and three dimensional numerical results for several standard test problems including a smooth Alfvén wave (to verify formal order of accuracy), shock tube problems (to test the shock-capturing ability of the scheme), Orszag-Tang, and cloud shock interactions. These results assert the robustness and verify the high-order of accuracy of the proposed scheme.
\end{abstract}

Keywords: magnetohydrodynamics; finite difference WENO; Lax-Wendroff; constrained transport; positivity preserving; high order

Email addresses: christli@msu.edu (Andrew J. Christlieb), fengxia2@msu.edu (Xiao Feng), seal@usna.edu

(David C. Seal), tangq30rpi.edu (Qi Tang)

${ }^{1}$ Corresponding author 


\section{Introduction}

The ideal magnetohydrodynamic (MHD) equations model the dynamics of a quasi-neutral, perfectly conducting plasma [1. A vast range of areas including astrophysics and laboratory plasmas can be modeled with this system. Among other methods, various high-order numerical schemes based on the essentially non-oscillatory (ENO) 2] as well as the weighted ENO (WENO) reconstruction technique [3, 4, 5, 6, 7, 8, 9, have been applied successfully to ideal MHD in the past two decades. These high order schemes are capable of resolving complex features such as shocks and turbulences using fewer grid points than low-order schemes for the same level of error, as is common with many high-order shock-capturing schemes.

It often happens in large-scale MHD simulations that the complex features are concentrated in a small portion of the simulation domain. Adaptive mesh refinement (AMR) is a technique that is designed for treating such locality of complexity in hydrodynamics and magnetohydrodynamics. One of the chief difficulties with implementing high order schemes within an AMR framework is that boundary conditions for the refined region need to be specified in a consistent manner 10. This becomes difficult for multistage RK methods, because high order solutions cannot be found if one simply uses high-order interpolated values (in time) at the ghost points that are required for the intermediate stages of the method. Preliminary work that combines WENO spatial discretizations with strong stability preserving Runge-Kutta (SSP-RK) time-stepping is conducted in [11, 12, and very recent work makes use of curvilinear grids to extend finite difference methods to problems with geometry [9. However, the authors in 9] use global time steps (which precludes the possibility of introducing local time stepping), and perhaps more troublesome, they drop mass conservation for their framework to work.

In choosing building blocks for AMR code, it has been argued that single-stage, single-step methods are advantageous [13, 14, partly because fewer synchronizations are needed per step than multistage RK methods. The fact that single-stage, single-step methods do not have an issue with these synchronizations is possibly one of the reasons they have gained much attention in the past two decades, and one reason we are choosing to pursue these methods. Broadly construed, these methods are based on Lax and Wendroff's original idea of using the Cauchy-Kovalevskaya procedure to convert temporal derivatives into spatial derivatives in order to define a numerical method [15. Notable highorder single-stage, single-step methods include the Arbitrary DERivative (ADER) methods [16, 17, the Lax-Wendroff finite difference WENO methods [18, the Lax-Wendroff discontinuous Galerkin (DG) methods [19] and space-time schemes applied directly to second-order wave equations [20, 21. Of the three classes of high-order methods based upon Lax-Wendroff time stepping, only the ADER methods have been applied to magnetohydrodynamics [14, 7, 22, whereas similar investigations have not been done for the other classes. An additional advantage that single-stage single-step Taylor methods offer is their low-storage opportunities. This requires care, because these methods can easily end up requiring the same amount of storage as their equivalent RK counterpart (e.g., if each time derivative is stored in order to reduce coding complexity).

The current work is based on the Taylor discretization of the Picard integral formulation of the finite difference WENO (PIF-WENO) method [23. Compared with other WENO methods that use Lax-Wendroff time discretizations [18, our method has the advantage that its focus is on constructing high-order Taylor expansions of the fluxes (which are used to define a conservative method through WENO reconstruction) as opposed to the conserved variables. This allows, for example, the adaptation of a positivity-preserving limiter, which we describe in this document.

An important issue in simulations of MHD systems is the controlling of the divergence error of the magnetic field, since numerical schemes based on the transport equations alone will, in general, accumulate errors in the divergence of the magnetic field. Failure to address this issue creates an unphysical force parallel to the magnetic field [24], and if this is not taken care of, it will often lead to failure of the simulation code. Popular techniques used to solve this problem include (1) the nonconservative eight-wave method [25], (2) the projection method [24], (3) the hyperbolic divergence cleaning method [26, and (4) the various constrained transport methods [27, 28, 8, 29, 30, 31, 32, 33, 34. Tóth conducts an extensive survey in [35. 
The current paper uses the unstaggered constrained transport framework proposed by Rossmanith [34]. This framework evolves a vector potential that sits on the same mesh as the conserved quantities. This vector potential to correct the magnetic field. Historically, the term "constrained transport" has been used to refer to a class of methods that incorporates the divergence-free condition into the discretization of the transport equation of the magnetic field, often done in a way that can be interpreted as maintaining an electric field on a staggered mesh [30, 35. Some authors actually still distinguish between this type of "constrained transport" and the "vector potential" approach [36]. However, as is well-known, evolving a vector potential is conceptually equivalent to evolving an electric field. The unstaggered approach has the added benefit of ease for potential embedding in an AMR framework.

An important piece in any vector-potential based constrained transport method is the discretization of the evolution equation of the vector potential. This evolution equation is a nonconservative weakly hyperbolic system, and it can be treated numerically from this viewpoint [33. An alternative approach is to view it as a modified system of Hamilton-Jacobi equations 8 . The current work adopts the latter approach, and uses a method inspired by a Lax-Wendroff numerical scheme for Hamilton-Jacobi equations that was proposed in [37]. The artificial resistivity terms used in [8] are adapted into the present single-stage, single-step scheme.

One further challenge for numerical simulations of MHD is that of retaining the positivity of the density and pressure. This is critical when the thermal pressure takes up only a small portion of the total energy, i.e., when the $\beta$ value is small. Almost all positivity-preserving methods exploit the presumed positivity-preserving property of certain low-order schemes, such as the Lax-Friedrichs scheme. Whereas earlier methods often rely on switching between high- and low-order updates 38, 39, more recent work tends to use combinations of the two. Examples include the work of Balsara [40] and Cheng et al. 41, which limit the conserved quantities at certain nodal points, and the work of Christlieb et al. [42, which combines high- and low-order fluxes through a single free parameter at each flux interface. In the current work, we adopt an approach similar to that used in [42]. This approach seeks a suitable convex combination of the high- and low-order fluxes at each cell at each time step. This flux limiter we adopt is an adaptation of the maximum principle preserving (MPP) flux limiter 43] (that has its roots in flux corrected transport schemes [44, 45]) for the purposes of retaining positivity of the density and pressure. We note that positivity-preserving has also been investigated for hydrodynamics. The limiters mentioned in this paragraph can be viewed as generalizations of limiters that have been applied to Euler's equations 46, 47, 48.

The outline of the paper is as follows. We first review the constrained transport framework in Section 2, Then in Section 3, we give a brief review of the Taylor-discretization PIF-WENO method, which we use for updating the conserved quantities. In Section 4 , we describe our method for evolving the vector potential, and the positivity-preserving limiter is described in Section 5 . In Section 6, we show the high-order accuracy and robustness of our method through a series of test problems. The conclusion and future work is given in Section 7

\section{A constrained transport framework}

In this section we review the unstaggered constrained transport framework that was proposed in 34. In conservation form, the ideal MHD equations are given by

$$
\partial_{t}\left[\begin{array}{c}
\rho \\
\rho \mathbf{u} \\
\mathcal{E} \\
\mathbf{B}
\end{array}\right]+\nabla \cdot\left[\begin{array}{c}
\rho \mathbf{u} \\
\rho \mathbf{u} \otimes \mathbf{u}+\left(p+\frac{1}{2}\|\mathbf{B}\|^{2}\right) \mathbb{I}-\mathbf{B} \otimes \mathbf{B} \\
\mathbf{u}\left(\mathcal{E}+p+\frac{1}{2}\|\mathbf{B}\|^{2}\right)-\mathbf{B}(\mathbf{u} \cdot \mathbf{B}) \\
\mathbf{u} \otimes \mathbf{B}-\mathbf{B} \otimes \mathbf{u} \\
\nabla \cdot \mathbf{B}=0
\end{array}\right]=0
$$

where $\rho$ is the mass density, $\rho \mathbf{u}$ is the momentum, $\mathcal{E}$ is the total energy, $p$ is the thermal pressure, $\|\cdot\|$ is the Euclidean vector norm, $\gamma=5 / 3$ is the ideal gas constant, and the pressure satisfies the 
equation of state

$$
\mathcal{E}=\frac{p}{\gamma-1}+\frac{\rho\|\mathbf{u}\|^{2}}{2}+\frac{\|\mathbf{B}\|^{2}}{2} .
$$

Equation (1) together with Equation (3) form a hyperbolic system of conservation laws. The eigendecomposition for this system is described in [49. For all the simulations in the current paper, we use the eigen-decomposition described in [50] (other decompositions have different scalings for the left and right eigenvectors). Equation (2) is not a constraint, but rather an involution [51, in the sense that if it is satisfied for the initial conditions, then it is satisfied for all later times. However, this condition must be explicitly enforced in the discretization of the system at each time step in order to maintain numerical stability.

The unstaggered constrained transport method in 34] makes use of the fact that because the magnetic field $\mathbf{B}$ is divergence-free, it admits a vector potential $\mathbf{A}$ that satisfies

$$
\mathbf{B}=\nabla \times \mathbf{A} .
$$

It is therefore possible to maintain, at the discrete level, the divergence-free property of $\mathbf{B}$ by evolving $\mathbf{A}$ alongside the conserved quantities, and correcting $\mathbf{B}$ to be the curl of $\mathbf{A}$.

The evolution equation for the magnetic potential can be derived by starting with Maxwell's equations. That is, if we start with Faraday's law (instead of starting with the ideal MHD equations), we have

$$
\partial_{t} \mathbf{B}=-\nabla \times \mathbf{E}
$$

Next, we approximate the electric field by Ohm's law for a perfect conductor

$$
\mathbf{E}=\mathbf{B} \times \mathbf{u}
$$

which yields the induction equation used for ideal MHD:

$$
\partial_{t} \mathbf{B}+\nabla \times(\mathbf{B} \times \mathbf{u})=0 .
$$

(After applying some appropriate vector identities, Eqn. 77) can be written in a conservative form.) By substituting (4) into (7), we obtain

$$
\nabla \times\left(\partial_{t} \mathbf{A}+(\nabla \times \mathbf{A}) \times \mathbf{u}\right)=0 .
$$

This implies there is a scalar function $\psi$ such that

$$
\partial_{t} \mathbf{A}+(\nabla \times \mathbf{A}) \times \mathbf{u}=-\nabla \psi .
$$

Different choices of $\psi$ in 99 correspond to different gauge conditions. It is noted in 32 that the Weyl gauge, which sets $\psi \equiv 0$, leads to stable numerical solutions. With the Weyl gauge, the evolution equation for magnetic potential 9 becomes

$$
\partial_{t} \mathbf{A}+(\nabla \times \mathbf{A}) \times \mathbf{u}=0 .
$$

In the constrained transport framework, both the conserved quantities $\mathbf{q}:=(\rho, \rho \mathbf{u}, \mathcal{E}, \mathbf{B})$ and the magnetic potential $\mathbf{A}$ are evolved. The time step from $t^{n}$ to $t^{n+1}$ proceeds as follows:

StepCT 1 Discretize (1) and update the conserved quantities

$$
\left(\rho^{n}, \rho \mathbf{u}^{n}, \mathcal{E}^{n}, \mathbf{B}^{n}\right) \rightsquigarrow\left(\rho^{n+1}, \rho \mathbf{u}^{n+1}, \mathcal{E}^{*}, \mathbf{B}^{*}\right),
$$

where $\mathcal{E}^{*}$ and $\mathbf{B}^{*}$ are subject to corrections described in Steps 3 and 4 below.

StepCT 2 Discretize 10 and update the magnetic potential: $\mathbf{A}^{n} \rightsquigarrow \mathbf{A}^{n+1}$. 
StepCT 3 Correct $\mathbf{B}$ to be the curl of $\mathbf{A}$ through

$$
\mathbf{B}^{n+1}=\nabla \times \mathbf{A} .
$$

Note that this step modifies the pressure.

StepCT 4 Modify the total energy so the pressure remains unchanged (see below).

In the current work, the discretization we use for (11) is the Taylor-discretization of the PIFWENO method [23]. This method is a single-stage single-step method for systems of hyperbolic conservation laws. We give a brief review of this method in Section 3

It is noted in 32 that Equation 10 is a weakly hyperbolic system that must be treated carefully in order to avoid numerical instabilities. Along the lines of our previous work in [8, we use a discretization that is inspired by numerical schemes for Hamilton-Jacobi equations, in order to be able to define non-oscillatory derivatives of the magnetic field. We present this discretization in Section 4 .

For certain problems, such as those involving low- $\beta$ plasma, negative density or pressure occur in numerical simulations, even when constrained transport is present. This requires the use of a positivity-preserving limiter [42, 48, 43]. This limiter preserves the positivity of density and pressure by modifying the numerical flux that evolves the conserved variables. We describe this limiter in detail in Section 5. When this limiter is used, we also apply the following fix to the pressure in StepCT 4 by modifying the total energy through

$$
\mathcal{E}^{n+1}=\mathcal{E}^{*}+\frac{1}{2}\left(\left\|\mathbf{B}^{n+1}\right\|^{2}-\left\|\mathbf{B}^{*}\right\|^{2}\right) .
$$

This energy "correction" keeps the pressure the same as before the magnetic field correction in 12 . While this clearly has the disadvantage of violating the conservation of energy, it is nonetheless needed in order to preserve the positivity of pressure (otherwise the modifications made to the magnetic field to obtain a divergence free property could potentially cause the pressure to become negative). This technique is explored in [28, 42, 35]. In the results presented in the current paper, we use the energy correction (13) if and only if the positivity-preserving limiter is turned on.

\section{The Taylor-discretization PIF-WENO method}

We now briefly review the Taylor-discretization Picard integral formulation weighted essentially non-oscillatory (PIF-WENO) method [23. This method applies to generic hyperbolic conservation laws in arbitrary dimensions, of which the ideal MHD equation is an example.

For the purpose of illustration, we present the method here for a $2 \mathrm{D}$ system. In $2 \mathrm{D}$, a hyperbolic conservation law takes the form

$$
\partial_{t} \mathbf{q}+\partial_{x} \mathbf{f}(\mathbf{q})+\partial_{y} \mathbf{g}(\mathbf{q})=0
$$

where $\mathbf{q}(t, x, y): \mathbb{R}^{+} \times \mathbb{R}^{2} \rightarrow \mathbb{R}^{m}$ is the vector of $m$ conserved variables, and $\mathbf{f}, \mathbf{g}: \mathbb{R}^{m} \rightarrow \mathbb{R}^{m}$ are the two components of the flux function. Formally integrating (14) in time from $t^{n}$ to $t^{n+1}$, one arrives at the Picard integral formulation of (14) given by

$$
\mathbf{q}^{n+1}=\mathbf{q}^{n}-\Delta t \partial_{x} \mathbf{F}^{n}(x, y)-\Delta t \partial_{y} \mathbf{G}^{n}(x, y),
$$

where the time-averaged fluxes $\mathbf{F}$ and $\mathbf{G}$ are defined as

$$
\mathbf{F}^{n}(x, y)=\frac{1}{\Delta t} \int_{t^{n}}^{t^{n+1}} \mathbf{f}(\mathbf{q}(t, x, y)) d t, \quad \text { and } \quad \mathbf{G}^{n}(x, y)=\frac{1}{\Delta t} \int_{t^{n}}^{t^{n+1}} \mathbf{g}(\mathbf{q}(t, x, y)) d t
$$


Given a domain $\Omega=\left[a_{x}, b_{x}\right] \times\left[a_{y}, b_{y}\right]$, the point-wise approximations $\mathbf{q}_{i, j}^{n} \approx \mathbf{q}\left(t^{n}, x_{i}, y_{j}\right)$ are placed at

$$
\begin{array}{lll}
x_{i}=a_{x}+\left(i-\frac{1}{2}\right) \Delta x, & \Delta x=\frac{b_{x}-a_{x}}{m_{x}}, & i \in\left\{1, \ldots, m_{x}\right\}, \\
y_{i}=a_{y}+\left(i-\frac{1}{2}\right) \Delta y, & \Delta y=\frac{b_{y}-a_{y}}{m_{y}}, & y \in\left\{1, \ldots, m_{y}\right\},
\end{array}
$$

and time $t=t^{n}$, where $m_{x}$ and $m_{y}$ are positive integers. The PIF-WENO scheme solves (14) by setting

$$
\mathbf{q}_{i, j}^{n+1}=\mathbf{q}_{i, j}^{n}-\frac{\Delta t}{\Delta x}\left(\hat{\mathbf{F}}_{i+1 / 2, j}^{n}-\hat{\mathbf{F}}_{i-1 / 2, j}^{n}\right)-\frac{\Delta t}{\Delta y}\left(\hat{\mathbf{G}}_{i, j+1 / 2}^{n}-\hat{\mathbf{G}}_{i, j-1 / 2}^{n}\right),
$$

where $\hat{\mathbf{F}}_{i \pm 1 / 2, j}$ and $\hat{\mathbf{G}}_{i, j \pm 1 / 2}$ are obtained by applying the classical WENO reconstruction to the time-averaged fluxes $\mathbf{F}_{i, j}^{n}$ and $\mathbf{G}_{i, j}^{n}$ instead of to the "frozen-in-time" fluxes, as is traditional in a method of lines (MOL) formulation. Here, $\mathbf{F}_{i, j}^{n}$ and $\mathbf{G}_{i, j}^{n}$ are approximated using a third-order Taylor expansion in time, followed by an application of Cauchy-Kovalevskaya procedure to replace the temporal derivatives with spatial derivatives. The resulting scheme on the conserved quantities is third-order accurate in time. We omit the details here and refer the reader to [23. Extensions to three dimensions follow by including a third component for the flux function.

\section{The evolution of the magnetic potential equation}

Recall that the evolution equation for the magnetic potential is defined in 10 . If we expand the curl and cross-product operator, we see that $\mathbf{A}$ satisfies

$$
\partial_{t} \mathbf{A}+N^{x} \partial_{x} \mathbf{A}+N^{y} \partial_{y} \mathbf{A}+N^{z} \partial_{z} \mathbf{A}=0,
$$

where

$$
N^{x}=\left[\begin{array}{ccc}
0 & -u^{y} & -u^{z} \\
0 & u^{x} & 0 \\
0 & 0 & u^{x}
\end{array}\right], \quad N^{y}=\left[\begin{array}{ccc}
u^{y} & 0 & 0 \\
-u^{x} & 0 & -u^{z} \\
0 & 0 & u^{y}
\end{array}\right], \quad N^{z}=\left[\begin{array}{ccc}
u^{z} & 0 & 0 \\
0 & u^{z} & 0 \\
-u^{x} & -u^{y} & 0
\end{array}\right]
$$

are matrices defined by components of the velocity field $\mathbf{u}=\left(u^{x}, u^{y}, u^{z}\right)$. The system 20 -21 has the following properties that we take into account when making a discretization:

1. This system is weakly hyperbolic. This means that all of the eigenvalues of 20 are real, but in contrast to being strictly hyperbolic, the system is not necessarily diagonalizable. While this weak hyperbolicity is an artifact of how the magnetic potential is evolved, it must be treated carefully to avoid creating numerical instabilities [32;

2. Each equation of this system can be viewed as a Hamilton-Jacobi equation with a source term 8 .

One consequence of this second observation is that we can use Hamilton-Jacobi technology in order to define magnetic fields that have non-oscillatory derivatives. That is, a single derivative of $\mathbf{B}$ is a second derivative of $\mathbf{A}$, in which case care need be taken in order to retain a non-oscillatory property.

In the next two subsections, we will describe how we discretize the system (20)-(21), with these two properties in mind. Before we do that, we make several remarks. The first is that in the discretization, A sits on the same mesh as the conserved quantities, hence the name "unstaggered constrained transport". The second is that the curl operator $\nabla \times$ is discretized in the same way as in 8 , which defines a divergence free magnetic field (at the discrete level). A final remark is that the discretization we present here is third-order accurate in time, which is in accordance with the discretization we use for the conserved quantities. 


\subsection{The $2 D$ magnetic potential equation}

We begin with the two-dimensional case, where the conserved quantities and the magnetic potential do not depend on $z$. In this case, the divergence-free condition is

$$
\nabla \cdot \mathbf{B}=\partial_{x} B^{x}+\partial_{y} B^{y}=0 .
$$

Thus $B^{z}$ has no impact on the divergence of $\mathbf{B}$. It therefore suffices to correct only the first two components of the magnetic field. A computationally cost-efficient way of doing this is to discard the first two components of the magnetic potential, evolve only the third component according to the evolution equation

$$
\partial_{t} A^{z}+u^{x} \partial_{x} A^{z}+u^{y} \partial_{y} A^{z}=0
$$

and for the magnetic field correction, only correct $B^{x}$ and $B^{y}$ through

$$
B^{x}=\partial_{y} A^{z} \quad \text { and } \quad B^{y}=-\partial_{x} A^{z} .
$$

This is equivalent to imposing $\partial_{z} A^{x}=0$ and $\partial_{z} A^{y}=0$ on the system (20)-(21), and skipping $B^{z}$ in the magnetic field correction in Eqn. (12).

Equation (23) has the favorable property of being strongly hyperbolic [34, in contrast to the weak hyperbolicity in the 3D case. This equation is also a Hamilton-Jacobi equation, with Hamilton principal function $A^{z}$, and Hamiltonian

$$
H\left(t, x, y, \partial_{x} A^{z}, \partial_{y} A^{z}\right)=u^{x}(t, x, y) \partial_{x} A^{z}+u^{y}(t, x, y) \partial_{y} A^{z} .
$$

It is therefore possible to discretize Equation (23) using a numerical scheme for Hamilton-Jacobi equations [8]. In line with the single-stage single-step theme of the proposed scheme, we use a method that is inspired by the Lax-Wendroff WENO schemes for Hamilton-Jacobi equations from 37.

A Lax-Wendroff WENO scheme for Hamilton-Jacobi equations is based on the Taylor expansion in time of the Hamilton principal function. In order to retain third-order accuracy in time, we require a total of three time derivatives of $A_{i, j}^{z}$ :

$$
A_{i, j}^{z n+1}=A_{i, j}^{z n}+\Delta t \partial_{t} A_{i, j}^{z n}+\frac{\Delta t^{2}}{2 !} \partial_{t}^{2} A_{i, j}^{z n}+\frac{\Delta t^{3}}{3 !} \partial_{t}^{3} A_{i, j}^{z n}
$$

where the temporal derivatives on the right-hand-side are computed in the following manner:

1. The first derivative is approximated by the Lax-Friedrichs type numerical Hamiltonian, with high-order reconstruction of $\partial_{x} A^{z}$ and $\partial_{y} A^{z}[52$. Namely, we define

$$
\begin{aligned}
\partial_{t} A_{i, j}^{z}:= & -\hat{H}\left(\partial_{x} A_{i, j}^{z-}, \partial_{x} A_{i, j}^{z+}, \partial_{y} A_{i, j}^{z-}, \partial_{y} A_{i, j}^{z+}\right) \\
= & -u_{i, j}^{x}\left(\frac{\partial_{x} A_{i, j}^{z-}+\partial_{x} A_{i, j}^{z+}}{2}\right)-u_{i, j}^{y}\left(\frac{\partial_{y} A_{i, j}^{z-}+\partial_{y} A_{i, j}^{z+}}{2}\right) \\
& +\alpha^{x}\left(\frac{\partial_{x} A_{i, j}^{z+}-\partial_{x} A_{i, j}^{z-}}{2}\right)+\alpha^{y}\left(\frac{\partial_{y} A_{i, j}^{z+}-\partial_{y} A_{i, j}^{z-}}{2}\right),
\end{aligned}
$$

where

$$
\alpha^{x}=\max _{i, j}\left|u_{i, j}^{x}\right| \quad \text { and } \quad \alpha^{y}=\max _{i, j}\left|u_{i, j}^{y}\right|
$$

are the maximum velocities taken over the entire grid, (i.e., the numerical flux is the global 
Lax-Friedrichs flux), and $\partial_{x} A_{i, j}^{z \pm}, \partial_{y} A_{i, j}^{z \pm}$ are defined by WENO reconstructions through

$$
\begin{aligned}
& \partial_{x} A_{i, j}^{z-}=\Phi_{\mathrm{WENO} 5}\left(\frac{\Delta_{x}^{+} A_{i-3, j}^{z}}{\Delta x}, \frac{\Delta_{x}^{+} A_{i-2, j}^{z}}{\Delta x}, \frac{\Delta_{x}^{+} A_{i-1, j}^{z}}{\Delta x}, \frac{\Delta_{x}^{+} A_{i, j}^{z}}{\Delta x}, \frac{\Delta_{x}^{+} A_{i+1, j}^{z}}{\Delta x},\right), \\
& \partial_{x} A_{i, j}^{z+}=\Phi_{\mathrm{WENO} 5}\left(\frac{\Delta_{x}^{+} A_{i+2, j}^{z}}{\Delta x}, \frac{\Delta_{x}^{+} A_{i+1, j}^{z}}{\Delta x}, \frac{\Delta_{x}^{+} A_{i, j}^{z}}{\Delta x}, \frac{\Delta_{x}^{+} A_{i-1, j}^{z}}{\Delta x}, \frac{\Delta_{x}^{+} A_{i-2, j}^{z}}{\Delta x},\right), \\
& \partial_{y} A_{i, j}^{z-}=\Phi_{\mathrm{WENO}}\left(\frac{\Delta_{y}^{+} A_{i, j-3}^{z}}{\Delta y}, \frac{\Delta_{y}^{+} A_{i, j-2}^{z}}{\Delta y}, \frac{\Delta_{y}^{+} A_{i, j-1}^{z}}{\Delta y}, \frac{\Delta_{y}^{+} A_{i, j}^{z}}{\Delta y}, \frac{\Delta_{y}^{+} A_{i, j+1}^{z}}{\Delta y},\right), \\
& \partial_{y} A_{i, j}^{z+}=\Phi_{\mathrm{WENO}}\left(\frac{\Delta_{y}^{+} A_{i, j+2}^{z}}{\Delta y}, \frac{\Delta_{y}^{+} A_{i, j+1}^{z}}{\Delta y}, \frac{\Delta_{y}^{+} A_{i, j}^{z}}{\Delta y}, \frac{\Delta_{y}^{+} A_{i, j-1}^{z}}{\Delta y}, \frac{\Delta_{y}^{+} A_{i, j-2}^{z}}{\Delta y},\right),
\end{aligned}
$$

and $\Delta_{x}^{+} A_{i, j}^{z}:=A_{i+1, j}^{z}-A_{i, j}^{z}$ and $\Delta_{y}^{+} A_{i, j}^{z}:=A_{i, j+1}^{z}-A_{i, j}^{z}$. The function $\Phi_{\text {WenO5 }}$ is the classical fifth-order WENO reconstruction whose coefficients can be found in many sources (e.g., [53, 54, 8] ). Note that this difference operator is designed for Hamilton-Jacobi problems, and does not produce the typical flux difference form that most hyperbolic solvers produce.

2. The higher derivatives $\partial_{t}^{2} A^{z}$ and $\partial_{t}^{3} A^{z}$ are converted into spatial derivatives by way of the Cauchy-Kovalevskaya procedure, and the resulting spatial derivatives are approximated using central differences. For example, $\partial_{t}^{2} A^{z}$ is converted into spatial derivatives by

$$
\begin{aligned}
\partial_{t}^{2} A^{z}= & \partial_{t}\left(\partial_{t} A^{z}\right) \\
= & \partial_{t}\left(-u^{x} \partial_{x} A^{z}-u^{y} \partial_{y} A^{z}\right) \\
= & -\partial_{t} u^{x} \partial_{x} A^{z}-u^{x} \partial_{t} \partial_{x} A^{z}-\partial_{t} u^{y} \partial_{y} A^{z}-u^{y} \partial_{t} \partial_{y} A^{z} \\
= & -\partial_{t} u^{x} \partial_{x} A^{z}-u^{x} \partial_{x}\left(\partial_{t} A^{z}\right)-\partial_{t} u^{y} \partial_{y} A^{z}-u^{y} \partial_{y}\left(\partial_{t} A^{z}\right) \\
= & -\partial_{t} u^{x} \partial_{x} A^{z}-u^{x} \partial_{x}\left(-u^{x} \partial_{x} A^{z}-u^{y} \partial_{y} A^{z}\right)-\partial_{t} u^{y} \partial_{y} A^{z}-u^{y} \partial_{y}\left(-u^{x} \partial_{x} A^{z}-u^{y} \partial_{y} A^{z}\right) \\
= & -\partial_{t} u^{x} \partial_{x} A^{z}-u^{x}\left(-\partial_{x} u^{x} \partial_{x} A^{z}-u^{x} \partial_{x}^{2} A^{z}-\partial_{x} u^{y} \partial_{y} A^{z}-u^{y} \partial_{x} \partial_{y} A^{z}\right) \\
& -\partial_{t} u^{y} \partial_{y} A^{z}-u^{y}\left(-\partial_{y} u^{x} \partial_{x} A^{z}-u^{x} \partial_{x} \partial_{y} A^{z}-\partial_{y} u^{y} \partial_{y} A^{z}-u^{y} \partial_{y}^{2} A^{z}\right),
\end{aligned}
$$

where

$$
\partial_{t} u^{x}=\partial_{t}\left(\frac{\rho u^{x}}{\rho}\right)=\frac{\partial_{t}\left(\rho u^{x}\right) \rho-\left(\rho u^{x}\right) \partial_{t} \rho}{\rho^{2}}
$$

and

$$
\partial_{t} u^{y}=\partial_{t}\left(\frac{\rho u^{y}}{\rho}\right)=\frac{\partial_{t}\left(\rho u^{y}\right) \rho-\left(\rho u^{y}\right) \partial_{t} \rho}{\rho^{2}},
$$

with $\partial_{t}\left(\rho u^{x}\right), \partial_{t}\left(\rho u^{y}\right)$, and $\partial_{t} \rho$ converted into spatial derivatives by way of (1). Note that we do not find it necessary to use a FD WENO discretization for $\rho_{t}$. There are a total of 49 distinct spatial derivatives that need to be approximated in (30)-32. Similar expressions exist for $\partial_{t}^{3} A^{z}$. These are done by using the central differencing formulae

$$
\begin{aligned}
\partial_{x} U_{i, j} & =\frac{-U_{i-1, j}+U_{i+1, j}}{2 \Delta x}, \\
\partial_{x}^{2} U_{i, j} & =\frac{U_{i-1, j}-2 U_{i, j}+U_{i+1, j}}{\Delta x^{2}}, \\
\partial_{x} \partial_{y} U_{i, j} & =\frac{U_{i-1, j-1}-U_{i-1, j+1}-U_{i+1, j-1}+U_{i+1, j+1}}{4 \Delta x \Delta y}, \\
\partial_{x}^{3} U_{i, j} & =\frac{-U_{i-2, j}+2 U_{i-1, j}-2 U_{i+1, j}+U_{i+2, j}}{2 \Delta x^{3}}, \\
\partial_{x}^{2} \partial_{y} U_{i, j} & =\frac{2 U_{i, j-1}-2 U_{i, j+1}-U_{i-1, j-1}+U_{i-1, j+1}-U_{i+1, j-1}+U_{i+1, j+1}}{2 \Delta x^{2} \Delta y},
\end{aligned}
$$




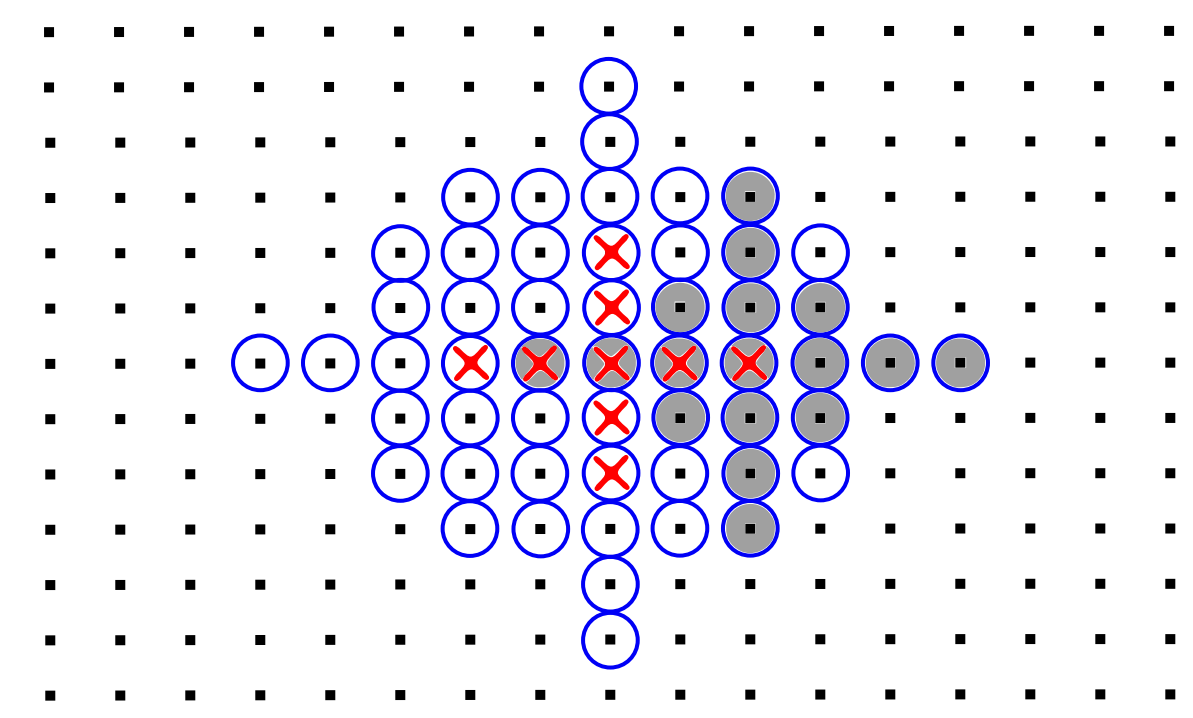

Figure 1: Stencil needed for magnetic potential. The values of $A^{z}$ at each "X" (save the centermost value) is needed to correct the value of $\mathbf{B}$ at the center. The shaded points are needed in Eqns. 29 and 333- 377 to compute the value of $A^{z}$ at the rightmost " $\times$ " point. Note that this stencil is contained in the stencil shown in [23, Fig. 2.

and similar ones for the $y$-, $y y$-, $x y y$ - and $y y y$-derivatives, where $U$ is any of $\rho, \rho u^{x}, \rho u^{y}, \rho u^{z}$, $\mathcal{E}, B^{x}, B^{y}, B^{z}$, or $A^{z}$.

Remark 1. In the current work, the magnetic field correction in Eqn. 24 is discretized to fourthorder accuracy with central difference formulas. For example, we define the $y$-derivative of $A^{z}$ to be

$$
B_{i, j}^{x}=\partial_{y} A^{z} \approx \frac{A_{i, j-2}^{z}-8 A_{i, j-1}^{z}+8 A_{i, j+1}^{z}-A_{i, j+2}^{z}}{12 \Delta y},
$$

and others similarly. Therefore in the discretization of equations 11 and 200, we aim for fourth-order accuracy in space. For the fluid equations in 11, we use the fifth-order accurate spatial discretization defined in 23. In the case of 20, Eqn. 26] is a fifth-order approximation to $\partial_{t} A^{z}\left(t^{n}, x_{i}, y_{j}\right)$ since the reconstructions 290 give, for example,

$$
\partial_{x} A_{i, j}^{z+}=\partial_{x} A^{z}\left(t^{n}, x_{i}, y_{j}\right)+\mathcal{O}\left(\Delta x^{5}\right)
$$

whereas Eqns. (33)- 37 are chosen so that Eqn. 267 is fourth-order accurate in space when $\Delta t=$ $\mathcal{O}(\Delta x)$. This fourth-order accuracy is verified in our numerical results.

Remark 2. In each dimension, the stencil needed to compute $\mathbf{B}$ from $\mathbf{A}$ is 5 points wide, and an

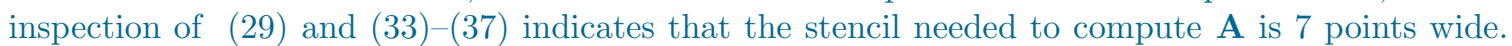
This results in a stencil that is 11 points wide in each dimension. A more careful analysis of (33)-(37) shows that this stencil is indeed contained within the stencil given by the PIF-WENO discretization of Eqn. 14 (which is sketched in Fig. 2 of [23). We present a sketch of the stencil required to discretize the magnetic potential in Fig. 1

We note that our discretization differs from that found in 37] in that we do not store any lower temporal derivatives for use in computation of higher temporal derivatives. That is, the method proposed in [37] stores the values of $\partial_{t} A^{z}$ computed in Equation (30), and makes use of the central differences of $\partial_{t} A^{z}$. Although that approach saves the trouble of expanding Eqn. (37), our approach has the following advantages that are in line with our goals: 
Minimal storage. Our approach avoids the necessity of storing the lower temporal derivatives (at the expense of a more complicated code). Note here that it would be not only $\partial_{t} A^{z}$ and $\partial_{t t} A^{z}$, but also the temporal derivatives of $\rho, \rho u^{x}, \rho u^{y}, \rho u^{z}, \mathcal{E}, B^{x}, B^{y}$, and $B^{z}$ that must be stored if the approach in [37] were to be taken. This would lead to a formidable amount of temporary storage when applied to the magnetic potential evolution equation in 3D;

Smaller stencils. The method in [37] would require a stencil that is 15 points wide in each dimension in order to provide the necessary A values for correcting the magnetic field. Our method only needs a stencil that is 11 points wide. This somewhat simplifies the future work of embedding the scheme into an AMR framework, but the biggest improvement is the reduction of temporary storage.

\subsection{The 3D magnetic potential equation}

The 3D magnetic potential equation (20)-(21) is weakly hyperbolic, and therefore special attention is needed. This can be treated by introducing artificial resistivity terms into the system [8, 32, 33]. To illustrate the technique, we consider the first row of the system which is

$$
\partial_{t} A^{x}-u^{y} \partial_{x} A^{y}-u^{z} \partial_{x} A^{z}+u^{y} \partial_{y} A^{x}+u^{z} \partial_{z} A^{x}=0 .
$$

A discretization of $\partial_{t} A^{x}$ in the spirit of (27) would give the following numerically problematic formulation

$$
\begin{aligned}
\partial_{t} A_{i, j, k}^{x}= & -u_{i, j, k}^{y}\left(\frac{\partial_{y} A_{i, j, k}^{x-}+\partial_{y} A_{i, j, k}^{x+}}{2}\right)-u_{i, j, k}^{z}\left(\frac{\partial_{z} A_{i, j, k}^{x-}+\partial_{z} A_{i, j, k}^{x+}}{2}\right) \\
& +\alpha^{y}\left(\frac{\partial_{y} A_{i, j, k}^{x+}-\partial_{y} A_{i, j, k}^{x-}}{2}\right)+\alpha^{z}\left(\frac{\partial_{z} A_{i, j, k}^{x+}-\partial_{z} A_{i, j, k}^{x-}}{2}\right) \\
& +u_{i, j, k}^{y}\left(\frac{\partial_{x} A_{i, j, k}^{y-}+\partial_{x} A_{i, j, k}^{y+}}{2}\right)+u_{i, j, k}^{z}\left(\frac{\partial_{x} A_{i, j, k}^{z-}+\partial_{x} A_{i, j, k}^{z+}}{2}\right)
\end{aligned}
$$

where

$$
\alpha^{y}=\max _{i, j, k}\left|u_{i, j, k}^{y}\right| \quad \text { and } \quad \alpha^{z}=\max _{i, j, k}\left|u_{i, j, k}^{z}\right|
$$

and $\partial_{y} A_{i, j, k}^{x-}, \partial_{y} A_{i, j, k}^{x+}, \partial_{z} A_{i, j, k}^{x-}, \partial_{z} A_{i, j, k}^{x+}, A_{x i, j, k}^{2-}, A_{x i, j, k}^{2+}, A_{x i, j, k}^{3-}$, and $A_{x i, j, k}^{3+}$ are reconstructed in a manner similar to 29 . The problem with (41) is that this formulation lacks numerical resistivity in the $x$-direction.

As is the case in [8], we find that the addition of an artificial resistivity term to 410 yields satisfactory numerical results. With the artificial resistivity term, the evolution equation 40 becomes

$$
\partial_{t} A^{x}-u^{y} \partial_{x} A^{y}-u^{z} \partial_{x} A^{z}+u^{y} \partial_{y} A^{x}+u^{z} \partial_{z} A^{x}=\epsilon^{x} \partial_{x}^{2} A^{x},
$$

where $\epsilon^{x}$ ideally satisfies

$$
\epsilon^{x}=\mathcal{O}\left(\Delta x^{6}\right) \text {, when } \partial_{x} A^{x} \text { is smooth, }
$$

and

$$
\epsilon^{x}=\mathcal{O}(\Delta x) \text {, when } \partial_{x} A^{x} \text { is non-smooth. }
$$

We define the artificial resistivity $\epsilon^{x}$ in the following manner. Define a smoothness indicator $\gamma^{x}$ for $\partial_{x} A^{x}$ as follows:

$$
\gamma_{i, j, k}^{x}=\left|\frac{a^{-}}{a^{-}+a^{+}}-\frac{1}{2}\right|,
$$

where

$$
a^{-}=\left(\epsilon+\left(\Delta x \partial_{x} A_{i, j, k}^{x-}\right)^{2}\right)^{-2} \quad \text { and } \quad a^{+}=\left(\epsilon+\left(\Delta x \partial_{x} A_{i, j, k}^{x+}\right)^{2}\right)^{-2} \text {. }
$$


Here, $\epsilon$ is a small positive number introduced to avoid dividing by a number close to 0 when the potential is smooth $\left(\epsilon=10^{-8}\right.$ in all our numerical simulations). Now we define $\epsilon^{x}$ to be

$$
\epsilon^{x}=2 \nu \gamma^{x} \frac{\Delta x^{2}}{\Delta t}
$$

where $\nu$ is a positive constant that controls the magnitude of the artificial resistivity. For a more detailed discussion of the weak hyperbolicity and the reasoning leading up to 48, we refer the reader to [32, 8. In [32, it is shown that $\nu$ has to be in the range of $[0,0.5]$ to maintain the stability up to CFL one for their finite volume method. Through numerical experimentation, we find that $\nu$ in the vicinity of 0.01 is sufficient to control potential oscillations in the magnetic field.

We thus use the following formulation as our discretization of 40 .

$$
\begin{aligned}
\partial_{t} A_{i, j, k}^{x}= & -u_{i, j, k}^{y}\left(\frac{\partial_{y} A_{i, j, k}^{x-}+\partial_{y} A_{i, j, k}^{x+}}{2}\right)-u_{i, j, k}^{z}\left(\frac{\partial_{z} A_{i, j, k}^{x-}+\partial_{z} A_{i, j, k}^{x+}}{2}\right) \\
& +\alpha^{y}\left(\frac{\partial_{y} A_{i, j, k}^{x+}-\partial_{y} A_{i, j, k}^{x-}}{2}\right)+\alpha^{z}\left(\frac{\partial_{z} A_{i, j, k}^{x+}-\partial_{z} A_{i, j, k}^{x-}}{2}\right) \\
& +u_{i, j, k}^{y}\left(\frac{\partial_{x} A_{i, j, k}^{y-}+\partial_{x} A_{i, j, k}^{y+}}{2}\right)+u_{i, j, k}^{z}\left(\frac{\partial_{x} A_{i, j, k}^{z-}+\partial_{x} A_{i, j, k}^{z+}}{2}\right) \\
& +2 \nu \gamma_{i, j, k}^{x}\left(\frac{A_{i-1, j, k}^{x}-2 A_{i, j, k}^{x}+A_{i+1, j, k}^{x}}{\Delta t}\right) .
\end{aligned}
$$

The discretization of $\partial_{t} A^{y}$ and $\partial_{t} A^{z}$ are similar and we omit them for brevity.

For our Lax-Wendroff formulation, it remains to discretize the higher temporal derivatives of the components of $\mathbf{A}$. We find it suffices to apply the same techniques as in Section 4.1 for $\partial_{t}^{2} A^{z}$ and $\partial_{t}^{3} A^{z}$. Namely, we convert all the temporal derivatives to spatial derivatives via the CauchyKovalevskaya procedure, and approximate the resulting spatial derivatives with central differences. We note that the artificial resistivity term is only added to the first temporal derivative.

\section{Positivity preservation}

The scheme presented thus far can be applied to a large class of problems. However, for problems where the plasma density or pressure are near zero, Gibb's phenomenon can cause these values to become negative, and hence the numerical simulation will instantly fail. As a final ingredient to the solver, we introduce an additional option for retaining positivity of the solution. Given the lack of number of degrees of freedom, this limiter comes at the expense of energy conservation, but these regions only occur in small areas where the density or pressure become negative.

A positivity-preserving scheme can be constructed by modifying the fluxes $\hat{\mathbf{F}}$ and $\hat{\mathbf{G}}$ in $(19)$. Let $\hat{\mathbf{f}}_{i+1 / 2, j}$ and $\hat{\mathbf{g}}_{i, j+1 / 2}$ be the (global) Lax-Friedrichs fluxes defined by

$$
\begin{aligned}
\hat{\mathbf{f}}_{i+1 / 2, j} & =\frac{1}{2}\left(\mathbf{f}\left(\mathbf{q}_{i+1, j}^{n}\right)+\mathbf{f}\left(\mathbf{q}_{i, j}^{n}\right)-\alpha^{x}\left(\mathbf{q}_{i+1, j}^{n}-\mathbf{q}_{i, j}^{n}\right)\right), \\
\hat{\mathbf{g}}_{i, j+1 / 2} & =\frac{1}{2}\left(\mathbf{g}\left(\mathbf{q}_{i, j+1}^{n}\right)+\mathbf{g}\left(\mathbf{q}_{i, j}^{n}\right)-\alpha^{y}\left(\mathbf{q}_{i, j+1}^{n}-\mathbf{q}_{i, j}^{n}\right)\right),
\end{aligned}
$$

where $\alpha^{x}$ and $\alpha^{y}$ are the maximal wave speeds in the $x$ and $y$ directions, respectively. This type of formulation is commonly referred to as Lax-Friedrich's flux splitting in the ENO/WENO literature 55, 56, 3.

The update of $\mathbf{q}$ using these Lax-Friedrichs fluxes is

$$
\mathbf{q}_{i, j}^{\mathrm{LF}}=\mathbf{q}_{i, j}^{n}-\frac{\Delta t}{\Delta x}\left(\hat{\mathbf{f}}_{i+1 / 2, j}^{n}-\hat{\mathbf{f}}_{i-1 / 2, j}^{n}\right)-\frac{\Delta t}{\Delta y}\left(\hat{\mathbf{g}}_{i, j+1 / 2}^{n}-\hat{\mathbf{g}}_{i, j-1 / 2}^{n}\right) .
$$


For an 8-component state vector $\mathbf{q}=(\rho, \rho \mathbf{u}, \mathcal{E}, \mathbf{B})$, we also introduce the notation $\rho(\mathbf{q})$ and $p(\mathbf{q})$ to represent the density and the thermal pressure of $\mathbf{q}$. Also, let $\epsilon_{\rho}$ and $\epsilon_{p}$ be small positive numbers.

The following claim was conjectured in [41].

Claim 1. If $\rho\left(\mathbf{q}_{i, j}^{n}\right)>\epsilon_{\rho}$ and $p\left(\mathbf{q}_{i, j}^{n}\right)>\epsilon_{p}$ for all $i, j$, and the CFL number is less than or equal to 0.5 , we then have $\rho\left(\mathbf{q}_{i, j}^{L F}\right)>\epsilon_{\rho}$ and $p\left(\mathbf{q}_{i, j}^{L F}\right)>\epsilon_{p}$ for all $i, j$.

Though not proven, this claim is verified in [41] using a fairly large number of random values of q. Our positivity limiter assumes this claim is true. However, as is noted in [41, if a different flux can be found such that it satisfies a property similar to that of the Lax-Friedrichs fluxes stated in Claim 1, we can then use these different fluxes in place of $\hat{\mathbf{f}}_{i+1 / 2, j}$ and $\hat{\mathbf{g}}_{i, j+1 / 2}$ in the construction of our positivity limiter.

The modified fluxes take the form

$$
\begin{aligned}
\tilde{\mathbf{F}}_{i+1 / 2, j} & =\theta_{i+1 / 2, j}\left(\hat{\mathbf{F}}_{i+1 / 2, j}-\hat{\mathbf{f}}_{i+1 / 2, j}\right)+\hat{\mathbf{f}}_{i+1 / 2, j}, \\
\tilde{\mathbf{G}}_{i, j+1 / 2} & =\theta_{i, j+1 / 2}\left(\hat{\mathbf{G}}_{i, j+1 / 2}-\hat{\mathbf{g}}_{i, j+1 / 2}\right)+\hat{\mathbf{g}}_{i, j+1 / 2},
\end{aligned}
$$

where $\theta_{i+1 / 2, j}$ and $\theta_{i, j+1 / 2}$ are chosen such that

- $0 \leq \theta_{i+1 / 2, j} \leq 1,0 \leq \theta_{i, j+1 / 2} \leq 1$,

- the update 19 with $\hat{\mathbf{F}}$ and $\hat{\mathbf{G}}$ replaced by the modified fluxes $\tilde{\mathbf{F}}$ and $\tilde{\mathbf{G}}$ defined in $(52)-(53)$ yields positive density and pressure, and

- while subject to the positivity requirement just stated, $\theta_{i+1 / 2, j}$ and $\theta_{i, j+1 / 2}$ should be as close to 1 as possible, so that the high-order fluxes are used in regions where violation of positivity is unlikely to happen.

Following [42, we choose each $\theta$ in a series of two steps:

Step- $\theta$ (i) For each $i, j$, find "large" candidate limiting parameters $\Lambda_{L, I_{i, j}}, \Lambda_{R, I_{i, j}}, \Lambda_{D, I_{i, j}}$, and $\Lambda_{U, I_{i, j}} \in[0,1]$ such that for all

$$
\left(\theta_{L}, \theta_{R}, \theta_{D}, \theta_{U}\right) \in\left[0, \Lambda_{L, I_{i, j}}\right] \times\left[0, \Lambda_{R, I_{i, j}}\right] \times\left[0, \Lambda_{D, I_{i, j}}\right] \times\left[0, \Lambda_{U, I_{i, j}}\right],
$$

the update defined by

$$
\begin{aligned}
\mathbf{q}:= & \mathbf{q}_{i, j}^{n}-\frac{\Delta t}{\Delta x}\left(\left(\theta_{R}\left(\hat{\mathbf{F}}_{i+1 / 2, j}-\hat{\mathbf{f}}_{i+1 / 2, j}\right)+\hat{\mathbf{f}}_{i+1 / 2, j}\right)-\left(\theta_{L}\left(\hat{\mathbf{F}}_{i-1 / 2, j}-\hat{\mathbf{f}}_{i-1 / 2, j}\right)+\hat{\mathbf{f}}_{i-1 / 2, j}\right)\right) \\
& -\frac{\Delta t}{\Delta y}\left(\left(\theta_{U}\left(\hat{\mathbf{G}}_{i, j+1 / 2}-\hat{\mathbf{f}}_{i, j+1 / 2}\right)+\hat{\mathbf{f}}_{i, j+1 / 2}\right)-\left(\theta_{D}\left(\hat{\mathbf{F}}_{i, j-1 / 2}-\hat{\mathbf{f}}_{i, j-1 / 2}\right)+\hat{\mathbf{f}}_{i, j-1 / 2}\right)\right)
\end{aligned}
$$

satisfies $\rho(\mathbf{q})>\epsilon_{\rho}$ and $p(\mathbf{q})>\epsilon_{p}$;

Step- $\theta$ (ii) For each $i, j$, set $\theta_{i+1 / 2, j}:=\min \left\{\Lambda_{R, I_{i, j}}, \Lambda_{L, I_{i+1, j}}\right\}$ and $\theta_{i, j+1 / 2}:=\min \left\{\Lambda_{U, I_{i, j}}, \Lambda_{D, I_{i, j+1}}\right\}$.

We note that with $\mathbf{q}_{i, j}^{n}, \hat{\mathbf{F}}, \hat{\mathbf{f}}, \hat{\mathbf{G}}$, and $\hat{\mathbf{g}}$ already computed, Equation 55 expresses the update $\mathbf{q}$ as an affine function of $\theta_{L}, \theta_{R}, \theta_{D}, \theta_{U}$, which, by abuse of notation, is denoted by $\mathbf{q}\left(\theta_{L}, \theta_{R}, \theta_{D}, \theta_{U}\right)$. The coefficients of the $\theta$ 's in $\mathbf{q}\left(\theta_{L}, \theta_{R}, \theta_{D}, \theta_{U}\right)$ are denoted by $\mathbf{C}_{L}, \mathbf{C}_{R}, \mathbf{C}_{D}$, and $\mathbf{C}_{U}$. Thus we have

$$
\begin{array}{rlrl}
\mathbf{C}_{L} & =\frac{\Delta t}{\Delta x}\left(\hat{\mathbf{F}}_{i-1 / 2, j}-\hat{\mathbf{f}}_{i-1 / 2, j}\right), & \mathbf{C}_{R} & =-\frac{\Delta t}{\Delta x}\left(\hat{\mathbf{F}}_{i+1 / 2, j}-\hat{\mathbf{f}}_{i+1 / 2, j}\right), \\
\mathbf{C}_{D} & =\frac{\Delta t}{\Delta y}\left(\hat{\mathbf{G}}_{i, j-1 / 2}-\hat{\mathbf{g}}_{i, j-1 / 2}\right), & \mathbf{C}_{U}=-\frac{\Delta t}{\Delta y}\left(\hat{\mathbf{G}}_{i, j+1 / 2}-\hat{\mathbf{g}}_{i, j+1 / 2}\right),
\end{array}
$$

and, with (51) in mind, the limited solution is

$$
\mathbf{q}\left(\theta_{L}, \theta_{R}, \theta_{D}, \theta_{U}\right)=\mathbf{q}_{i, j}^{\mathrm{LF}}+\mathbf{C}_{L} \theta_{L}+\mathbf{C}_{R} \theta_{R}+\mathbf{C}_{D} \theta_{D}+\mathbf{C}_{U} \theta_{U}
$$

With this notation, Step- $\theta$ (ii) reduces to solving the following problem for each $i, j$ : 
Problem 1. Given constant real vectors $\mathbf{C}_{L}, \mathbf{C}_{R}, \mathbf{C}_{D}$ and $\mathbf{C}_{U}$, and constant 8-component state vector $\mathbf{q}_{i, j}^{L F}$ such that $\rho\left(\mathbf{q}_{i, j}^{L F}\right)>\epsilon_{\rho}$ and $p\left(\mathbf{q}_{i, j}^{L F}\right)>\epsilon_{p}$, find $\left(\Lambda_{L, I_{i, j}}, \Lambda_{R, I_{i, j}}, \Lambda_{D, I_{i, j}}, \Lambda_{U, I_{i, j}}\right)$ in $[0,1] \times$ $[0,1] \times[0,1] \times[0,1]$ such that for all $\left(\theta_{L}, \theta_{R}, \theta_{D}, \theta_{U}\right)$ in $\left[0, \Lambda_{L, I_{i, j}}\right] \times\left[0, \Lambda_{R, I_{i, j}}\right] \times\left[0, \Lambda_{D, I_{i, j}}\right] \times\left[0, \Lambda_{U, I_{i, j}}\right]$, the expression

$$
\mathbf{q}\left(\theta_{L}, \theta_{R}, \theta_{D}, \theta_{U}\right)=\mathbf{q}_{i, j}^{L F}+\mathbf{C}_{L} \theta_{L}+\mathbf{C}_{R} \theta_{R}+\mathbf{C}_{D} \theta_{D}+\mathbf{C}_{U} \theta_{U}
$$

satisfies

$$
\rho\left(\mathbf{q}\left(\theta_{L}, \theta_{R}, \theta_{D}, \theta_{U}\right)\right)>\epsilon_{\rho}
$$

and

$$
p\left(\mathbf{q}\left(\theta_{L}, \theta_{R}, \theta_{D}, \theta_{U}\right)\right)>\epsilon_{p} .
$$

The region $\left[0, \Lambda_{L, I_{i, j}}\right] \times\left[0, \Lambda_{R, I_{i, j}}\right] \times\left[0, \Lambda_{D, I_{i, j}}\right] \times\left[0, \Lambda_{U, I_{i, j}}\right]$ should be "as big as possible".

We note the following fact.

Claim 2. The sets $S_{\rho}$ and $S$ defined by

$$
S_{\rho}=\left\{\left(\theta_{L}, \theta_{R}, \theta_{D}, \theta_{U}\right) \in[0,1]^{4} \mid \rho\left(\mathbf{q}\left(\theta_{L}, \theta_{R}, \theta_{D}, \theta_{U}\right)\right)>\epsilon_{\rho}\right\}
$$

and

$$
S=\left\{\left(\theta_{L}, \theta_{R}, \theta_{D}, \theta_{U}\right) \in[0,1]^{4} \mid \rho\left(\mathbf{q}\left(\theta_{L}, \theta_{R}, \theta_{D}, \theta_{U}\right)\right)>\epsilon_{\rho} \text { and } p\left(\mathbf{q}\left(\theta_{L}, \theta_{R}, \theta_{D}, \theta_{U}\right)\right)>\epsilon_{p}\right\}
$$

are both convex.

Proof. Since $\rho$ is a linear function of $\mathbf{q}$ and $\mathbf{q}$ is a affine function of $\left(\theta_{L}, \theta_{R}, \theta_{D}, \theta_{U}\right)$, we see that $\rho\left(\mathbf{q}\left(\theta_{L}, \theta_{R}, \theta_{D}, \theta_{U}\right)\right)$ is an affine function of $\left(\theta_{L}, \theta_{R}, \theta_{D}, \theta_{U}\right)$. Thus $S_{\rho}$ is the part of $[0,1]^{4}$ that lies on one side of a hyperplane. This shows $S_{\rho}$ is convex.

To see the convexity of $S$, note that by the equation of state 3 , the pressure $p$ is a concave function of the components of $\mathbf{q}$, whenever $\rho>0$. Combined with the fact that $\mathbf{q}\left(\theta_{L}, \theta_{R}, \theta_{D}, \theta_{U}\right)$ is an affine function of $\left(\theta_{L}, \theta_{R}, \theta_{D}, \theta_{U}\right)$, we see that $p\left(\mathbf{q}\left(\theta_{L}, \theta_{R}, \theta_{D}, \theta_{U}\right)\right)$ is a concave function of $\left(\theta_{L}, \theta_{R}, \theta_{D}, \theta_{U}\right)$, if $\rho\left(\mathbf{q}\left(\theta_{L}, \theta_{R}, \theta_{D}, \theta_{U}\right)\right)>0$. This shows the convexity of $S_{p}$.

Note that Problem 1 is not well-defined, since the notion of "big" is not defined. The algorithm we are about to describe gives a solution that is satisfactory, in the sense that this algorithm yields a positivity-preserving limiter that behaves well in our numerical tests.

We now describe this algorithm. The first step of this algorithm is to find a "big" rectangular subset $R_{\rho}:=\left[0, \Lambda_{L}^{\rho}\right] \times\left[0, \Lambda_{R}^{\rho}\right] \times\left[0, \Lambda_{D}^{\rho}\right] \times\left[0, \Lambda_{U}^{\rho}\right]$ of $S_{\rho}$. The $\Lambda^{\rho}$ 's are computed by

$$
\Lambda_{\mathcal{I}}^{\rho}=\left\{\begin{array}{ll}
\min \left\{1, \frac{\rho\left(\mathbf{q}_{i, j}^{\mathrm{LF}}\right)-\epsilon_{\rho}}{\epsilon+\sum_{\mathcal{J},}\left|C_{\mathcal{J}}^{(1)}\right|}\right. \\
C_{\mathcal{J}}^{(1)}<0
\end{array}\right\} \begin{aligned}
& \text { if } C_{\mathcal{I}}^{(1)}<0, \\
& \text { if } C_{\mathcal{I}}^{(1)} \geq 0,
\end{aligned}
$$

where $\epsilon$ is a small fixed positive number $\left(10^{-12}\right.$ in all our simulations) and the subscript letters $\mathcal{I}$ and $\mathcal{J}$ take values in $L, R, D$, and $U$. The value $C_{\mathcal{I}}^{(1)}$ denotes the first component of the $\mathbf{C}_{\mathcal{I}}$ vector.

The second step is to shrink this rectangular subset $R_{\rho}$ to fit into $S_{p}$. The vertices of $R_{\rho}$ are denoted by $\mathbf{A}^{k_{L}, k_{R}, k_{D}, k_{U}}$, where $k_{\mathcal{I}}=0$ or 1 , such that the $\mathcal{I}$-th component of $\mathbf{A}^{k_{L}, k_{R}, k_{D}, k_{U}}$ is

$$
A_{\mathcal{I}}^{k_{L}, k_{R}, k_{D}, k_{U}}= \begin{cases}\Lambda_{\mathcal{I}}^{\rho} & \text { if } k_{\mathcal{I}}=1 \\ 0 & \text { if } k_{\mathcal{I}}=0\end{cases}
$$


Now for each $\left(k_{L}, k_{R}, k_{D}, k_{U}\right)$, shrink $\mathbf{A}^{k_{L}, k_{R}, k_{D}, k_{U}}$ to get $\mathbf{B}^{k_{L}, k_{R}, k_{D}, k_{U}}$ in the following way. If $p\left(\mathbf{A}^{k_{L}, k_{R}, k_{D}, k_{U}}\right) \geq \epsilon_{p}$, set $\mathbf{B}^{k_{L}, k_{R}, k_{D}, k_{U}}=\mathbf{A}^{k_{L}, k_{R}, k_{D}, k_{U}}$. Otherwise, we solve for the smallest positive $r$ such that $p\left(r \mathbf{A}^{\bar{k}_{L}, k_{R}, k_{D}, k_{U}}\right) \geq \epsilon_{p}$, and set $\mathbf{B}^{k_{L}, k_{R}, k_{D}, k_{U}}=r \mathbf{A}^{k_{L}, k_{R}, k_{D}, k_{U}}$. In order to solve for this value, we apply a total of 10 iterations of the bisection method. A more efficient (approximate) solver could easily replace this step (e.g., a single step of the method of false position). Note that the different vertices $\mathbf{A}^{k_{L}, k_{R}, k_{D}, k_{U}}$ are in general shrunk by different factors $r$. Now we set

$$
\Lambda_{\mathcal{I}, I_{i, j}}=\min _{\substack{\left(k_{L}, k_{R}, k_{U}, k_{D}\right), k_{\mathcal{I}=1}}} B_{\mathcal{I}}^{k_{L}, k_{R}, k_{U}, k_{D}},
$$

where the subscript $\mathcal{I}$ indicates the $\mathcal{I}$-th component. Note that this is equivalent to finding a rectangular subset inside the convex polygon with vertices $\mathbf{B}^{k_{L}, k_{R}, k_{D}, k_{U}}, k_{\mathcal{I}}=0,1$.

This completes the description of our positivity-preserving limiter in $2 \mathrm{D}$. The $3 \mathrm{D}$ case is similar.

\section{Numerical results}

In this section, we present the results of numerical simulations using the proposed method. Unless otherwise specified, constrained transport is turned on, the gas constant $\gamma=5 / 3$, the CFL number is 0.5 , and for $3 \mathrm{D}$ simulations the artificial viscosity coefficient is $\nu=0.01$.

\subsection{Smooth Alfvén wave}

The smooth Alfvén wave problem is often used for convergence studies of numerical schemes for ideal MHD equations 32, 34, 35]. This problem is a one-dimensional problem (computed in multiple dimensions) that has a known smooth solution. In 1D, the initial conditions for this problem are

$$
\begin{aligned}
& \left(\rho, u^{x}, u^{y}, u^{z}, u^{z}, p, B^{x}, B^{y}, B^{z}\right)(0, x) \\
& \quad=(1,0,0.1 \sin (2 \pi x), 0.1 \cos (2 \pi x), 0.1,1,0.1 \sin (2 \pi x), 0.1 \cos (2 \pi x)) .
\end{aligned}
$$

The exact solution to 66 propagates with the Alfvén speed that is unity (i.e., $\mathbf{q}(t, x)=\mathbf{q}(0, x+t))$. The $2 \mathrm{D}$ and $3 \mathrm{D}$ smooth Alfvén wave problems are obtained from the $1 \mathrm{D}$ problem by rotating the direction of wave propagation.

\subsubsection{Smooth Alfvén wave: The 2D problem}

The $2 \mathrm{D}$ version of the smooth Alfvén wave problem is obtained by rotating the direction of propagation by an angle of $\phi$, so that the wave now propagates in direction $\mathbf{n}=\langle-\cos \phi,-\sin \phi, 0\rangle$. Identical to [32, 8], the computational domain we use is $[0,1 / \cos \phi] \times[0,1 / \sin \phi]$, where $\phi=$ $\tan ^{-1}(0.5)$. Periodic boundary conditions are applied on all four sides.

For this problem, we present numerical results with and without the energy correction. In Table 1. we observe the overall third-order accuracy of the method, and in Table 2, we refine $\Delta t$ faster than the mesh spacing as well as run the solution to a shorter final time in order to extract the spatial order of accuracy. For these test cases, we observe the predicted fourth-order accuracy in space. of convergence in space, third-order in time, and little difference between the results obtained with and without the energy correction turned on. When the flag for the positivity-preserving limiter is turned on in the code, we see identical results as without it, because this problem does not have density or pressure that is near zero. The choice of $m_{y}=2 m_{x}$ allows for $\Delta x=\Delta y$.

\subsubsection{Smooth Alfvén wave: The 3D problem} is

The setup we use here is the same as that used in [32], Section 6.2.1. The direction of propagation

$$
\mathbf{n}=\langle-\cos \phi \cos \theta,-\sin \phi \cos \theta, \sin \theta\rangle,
$$

and the computational domain is

$$
\left[0, \frac{1}{\cos \phi \cos \theta}\right] \times\left[0, \frac{1}{\sin \phi \cos \theta}\right] \times\left[0, \frac{1}{\sin \theta}\right],
$$


Table 1: 2D smooth Alfvén wave. Here, we show $L^{\infty}$-errors at a final time of $t=1.0$ for the solution with and without the energy "correction" step. Left two columns have the energy correction turned off, and the rightmost columns have the energy correction turned on. Because time is only discretized to third-order accuracy, we observe the predicted third-order accuracy of the solver here.

\begin{tabular}{|l|l||l|l|l|l||l|l|l|l|}
\hline Mesh & CFL & Error in B & Order & Error in $A^{z}$ & Order & Error in B & Order & Error in $A^{z}$ & Order \\
\hline \hline $32 \times 64$ & 0.5 & $3.842 \times 10^{-5}$ & - & $5.356 \times 10^{-6}$ & - & $3.848 \times 10^{-5}$ & - & $5.320 \times 10^{-6}$ & - \\
$64 \times 128$ & 0.5 & $4.940 \times 10^{-6}$ & 2.96 & $7.530 \times 10^{-7}$ & 2.83 & $4.938 \times 10^{-6}$ & 2.96 & $7.469 \times 10^{-7}$ & 2.83 \\
$128 \times 256$ & 0.5 & $6.324 \times 10^{-7}$ & 2.97 & $9.697 \times 10^{-8}$ & 2.96 & $6.318 \times 10^{-7}$ & 2.97 & $9.628 \times 10^{-8}$ & 2.96 \\
$256 \times 512$ & 0.5 & $8.020 \times 10^{-8}$ & 2.98 & $1.218 \times 10^{-8}$ & 2.99 & $8.009 \times 10^{-8}$ & 2.98 & $1.210 \times 10^{-8}$ & 2.99 \\
\hline \hline
\end{tabular}

Table 2: $2 \mathrm{D}$ smooth Alfvén wave. Here, we show $L^{\infty}$-errors at a short final time of $t=0.01$ for the solution with and without the energy "correction" step. Left two columns have the energy correction turned off, and the rightmost columns have the energy correction turned on. Here, we refine $\Delta t$ faster than $\Delta x$ in order to expose the spatial order of accuracy of the solver. Because we only use a fourth-order accurate spatial discretization for $\nabla \times \mathbf{A}$, we only observe fourth-order accuracy despite the fact that the fluid variables are discretized to fifth-order accuracy.

\begin{tabular}{|c|c|c|c|c|c|c|c|c|c|}
\hline Mesh & CFL & Error in $\mathbf{B}$ & Order & Error in $A^{z}$ & Order & Error in $\mathbf{B}$ & Order & Error in $A^{z}$ & Order \\
\hline $32 \times 64$ & 0.5 & $3.852 \times 10^{-6}$ & - & $1.078 \times 10^{-7}$ & - & $3.852 \times 10^{-6}$ & 一 & $1.078 \times 10^{-7}$ & 一 \\
\hline $64 \times 128$ & 0.25 & $2.356 \times 10^{-7}$ & 4.03 & $8.121 \times 10^{-9}$ & 3.73 & $2.356 \times 10^{-7}$ & 4.03 & $8.121 \times 10^{-9}$ & 3.73 \\
\hline $128 \times 256$ & 0.125 & $1.466 \times 10^{-8}$ & 4.01 & $5.190 \times 10^{-10}$ & 3.97 & $1.466 \times 10^{-8}$ & 4.01 & $5.190 \times 10^{-10}$ & 3.97 \\
\hline $256 \times 512$ & 0.0625 & $9.117 \times 10^{-10}$ & 4.01 & $3.291 \times 10^{-11}$ & 3.98 & $9.117 \times 10^{-10}$ & 4.01 & $3.291 \times 10^{-11}$ & 3.98 \\
\hline
\end{tabular}

where $\phi=\theta=\tan ^{-1}(0.5)$. Periodic boundary conditions are imposed on all directions.

We again seek to numerically investigate the spatial and temporal orders of accuracy, with and without the energy correction step. The errors in $\mathbf{B}$ and $\mathbf{A}$ are presented in Tables 34 Here we choose $m_{y}=m_{z}=2 m_{x}$ so that $\Delta x=\Delta y=\Delta z / \cos \theta$. Similar to the 2D case, we observe fourthorder of convergence in space, third-order in time, and little difference between the results obtained with and without the energy correction step turned on.

\subsection{D rotated shock tube problem}

Similar to the smooth Alfvén problems, the rotated shock tube problem is a 1D problem, with direction of wave propagation rotated a certain angle. The setup we use in the current work is the same as that in 8, Section 7.2, which we repeat here for completeness.

The initial conditions consist of a shock

$$
\left(\rho, u_{\perp}, u_{\|}, u^{z}, p, B_{\perp}, B_{\|}, B^{z}\right)= \begin{cases}(1,-0.4,0,0,1,0.75,1,0) & \text { if } \xi<0 \\ (0.2,-0.4,0,0,0.1,0.75,-1,0) & \text { if } \xi \geq 0\end{cases}
$$

Table 3: 3D smooth Alfvén wave. In this table we show the $L^{\infty}$-errors at a moderate time of $t=1.0$. In the left columns the positivity-preserving limiter is off, and the the positivity-preserving limiter (and the energy correction step) is turned on for the results in the right columns. Because time is discretized to third-order accuracy, the final method is formally only third-order accurate in time. In Table 4 we run the solver to a short final time in order to expose the spatial order of accuracy.

\begin{tabular}{|l|l||l|l|l|l||l|l|l|l|}
\hline Mesh & CFL & Error in B & Order & Error in A & Order & Error in B & Order & Error in A & Order \\
\hline \hline $16 \times 32 \times 32$ & 0.5 & $4.784 \times 10^{-4}$ & - & $5.116 \times 10^{-5}$ & - & $4.882 \times 10^{-4}$ & - & $5.176 \times 10^{-5}$ & - \\
$32 \times 64 \times 64$ & 0.5 & $2.452 \times 10^{-5}$ & 4.29 & $3.181 \times 10^{-6}$ & 4.01 & $2.485 \times 10^{-5}$ & 4.30 & $3.191 \times 10^{-6}$ & 4.02 \\
$64 \times 128 \times 128$ & 0.5 & $3.093 \times 10^{-6}$ & 2.99 & $4.612 \times 10^{-7}$ & 2.79 & $3.105 \times 10^{-6}$ & 3.00 & $4.621 \times 10^{-7}$ & 2.79 \\
$128 \times 256 \times 256$ & 0.5 & $3.969 \times 10^{-7}$ & 2.96 & $6.133 \times 10^{-8}$ & 2.91 & $3.977 \times 10^{-7}$ & 2.96 & $6.147 \times 10^{-8}$ & 2.91 \\
\hline \hline
\end{tabular}


Table 4: 3D smooth Alfvén wave. Here, we show the $L^{\infty}$-errors at a short final time of $t=0.01$. In addition, we refine $\Delta t$ faster than the mesh spacing in order to extract the spatial order of accuracy. Left two columns have the turned off, and the right two columns have the positivity-preserving limiter (as well as the correction step) turned on. The results are almost identical.

\begin{tabular}{|l|l||l|l|l|l||l|l|l|l|}
\hline Mesh & CFL & Error in B & Order & Error in A & Order & Error in B & Order & Error in A & Order \\
\hline \hline $16 \times 32 \times 32$ & 0.5 & $6.752 \times 10^{-5}$ & - & $5.715 \times 10^{-7}$ & - & $6.752 \times 10^{-5}$ & - & $5.715 \times 10^{-7}$ & - \\
$32 \times 64 \times 64$ & 0.25 & $4.280 \times 10^{-6}$ & 3.98 & $3.856 \times 10^{-8}$ & 3.89 & $4.280 \times 10^{-6}$ & 3.98 & $3.856 \times 10^{-8}$ & 3.89 \\
$64 \times 128 \times 128$ & 0.125 & $2.666 \times 10^{-7}$ & 4.00 & $2.613 \times 10^{-9}$ & 3.88 & $2.666 \times 10^{-7}$ & 4.00 & $2.613 \times 10^{-9}$ & 3.88 \\
$128 \times 256 \times 256$ & 0.0625 & $1.652 \times 10^{-8}$ & 4.01 & $1.711 \times 10^{-10}$ & 3.93 & $1.652 \times 10^{-8}$ & 4.01 & $1.712 \times 10^{-10}$ & 3.93 \\
\hline \hline
\end{tabular}

where $\xi=x \cos \phi+y \sin \phi$, and $u_{\perp}$ and $B_{\perp}$ are vector components perpendicular to the shock interface, and $u_{\|}$and $B_{\|}$are the vector components parallel to the shock interface. Namely

$$
\begin{array}{cl}
u^{x}=u_{\perp} \cos \phi-u_{\|} \sin \phi, & u^{y}=u_{\perp} \sin \phi+u_{\|} \cos \phi, \\
B^{x}=B_{\perp} \cos \phi-B_{\|} \sin \phi, & B^{y}=B_{\perp} \sin \phi+B_{\|} \cos \phi .
\end{array}
$$

The initial condition for magnetic potential is

$$
A^{z}(0, x, y)= \begin{cases}0.75 \eta-\xi & \text { if } \xi \leq 0 \\ 0.75 \eta+\xi & \text { if } \xi>0\end{cases}
$$

where $\eta=-x \sin \phi+y \cos \phi$.

The computational domain is $[-1.2,1.2] \times[-1,1]$ with a $180 \times 150$ mesh. The boundary conditions used are zeroth order extrapolation on the conserved quantities and first-order extrapolation on the magnetic potential. That is, we set the conserved quantities at the ghost points to be identical to the last value on the interior of the domain, and we define values for the magnetic potential at ghost points through repeated extrapolation of two point stencils starting with two interior points. On the top and bottom boundaries, the direction of extrapolation is parallel to the shock interface.

In Figure 2 we present results for the density of solutions computed using PIF-WENO with and without constrained-transport. We note that the contour plot of the solution obtained without constrained transport does not exhibit the unphysical wiggles as is the case in Fig 2(b) of 8]. However, as can be seen from the plots of the slice at $y=0$, unphysical oscillations appear in the PIF-WENO scheme that has constrained transport turned off. It is also clear from these plots that the constrained transport method we propose in the current work is able to suppress the unphysical oscillations satisfactorily. As a side note, we find it helps to use a global, as opposed to a local value for $\alpha$ in the Lax-Friedrichs flux splitting for the high-order WENO reconstruction in order to further reduce undesirable spurious oscillations.

\subsection{D Orszag-Tang vortex}

In this section, we investigate the Orszag-Tang vortex problem. A notable feature of this problem is that shocks and cortices emerge from smooth initial conditions as time evolves. This is a standard test problem for numerical schemes for MHD equations [8, 57, 34, 35, 58. The initial conditions are

$$
\rho=\gamma^{2}, \quad \mathbf{u}=(-\sin (y), \sin (x), 0), \quad \mathbf{B}=(-\sin (y), \sin (2 x), 0), \quad p=\gamma,
$$

with an initial magnetic potential of

$$
A^{z}(0, x, y)=0.5 \cos (2 x)+\cos (y) .
$$

The computational domain is $[0,2 \pi] \times[0,2 \pi]$, with double-periodic boundary conditions.

We present in Figure 4 the density contour plots at $t=0.5, t=2, t=3$, and $t=4$, computed by PIF-WENO with constrained transport on and positivity-preserving limiter on. 

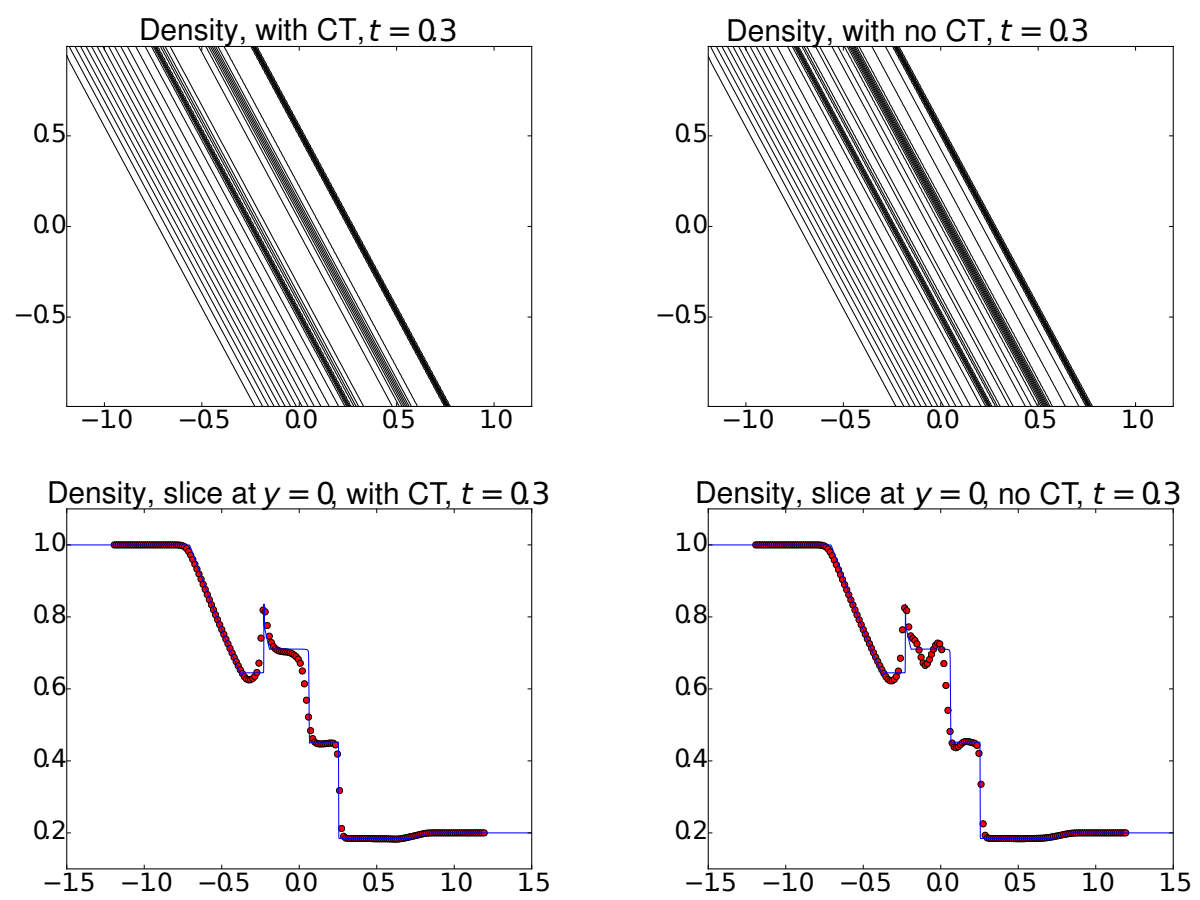

Figure 2: The rotated shock tube problem. Here, we compare the solver with (left panels) and without (right panels) constrained transport turned on. A uniform mesh of size $180 \times 150$ is used for both simulations. The angle of rotation for the initial conditions is $\phi=\tan ^{-1}(0.5)$, and 30 equally spaced contours ranging from the minumum to the maximum of each function are used for the top two panels. The contour plot for the solution without CT contains small wiggles that are much more pronounced when slices of the solution are sampled. To this end, a slice of the solution along $y=0$ is presented in the bottom two panels. Further evolution produces a solution that causes the code to fail in the case where CT is turned off. The positivity-preserving limiter is turned off in order to exercise the code. The solid lines in the bottom images are reference solutions that are computed by solving the equivalent $1 \mathrm{D}$ shock problem on a uniform mesh with 50,000 points with the fifth-order finite difference WENO method. 

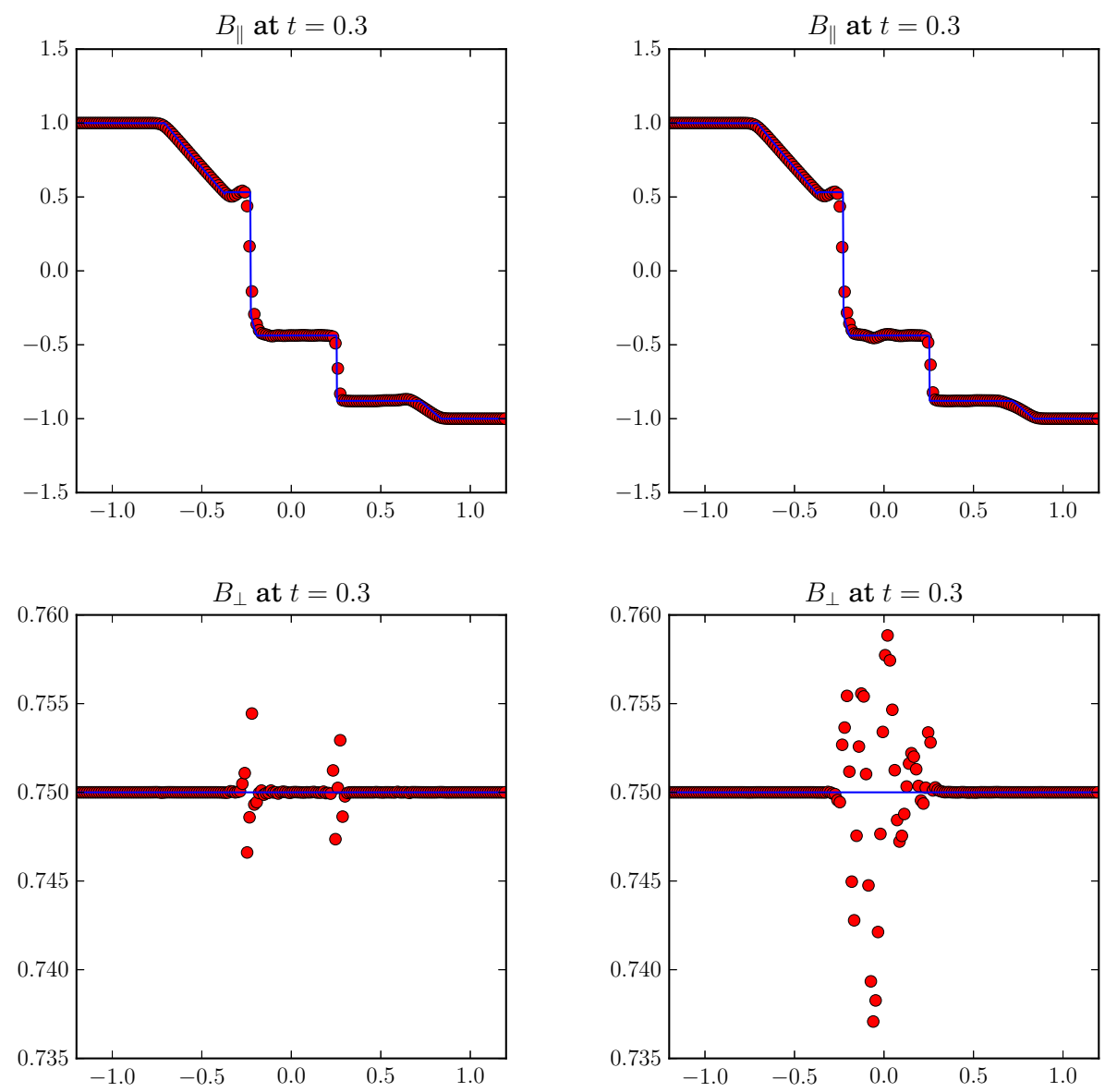

With Constrained Transport

No Constrained Transport

Figure 3: 2D rotated shock tube problem. The left panels have constrained transport turned on, and the right panels have constrained transport turned off. Components of the magnetic field at $t=0.3$ along the slice $y=0$. The mesh size is $180 \times 150$. The positivity-preserving limiter is turned off in order to exercise the code. Each solid line is the reference solution described in Fig. 22 We observe that constrained transport (with the Hamilton-Jacobi solver) allows us to numerically compute magnetic fields with far fewer oscillations than would otherwise be obtainable. 


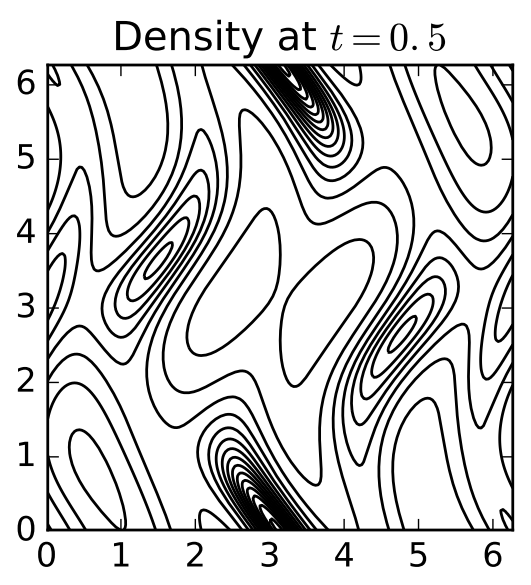

(a)

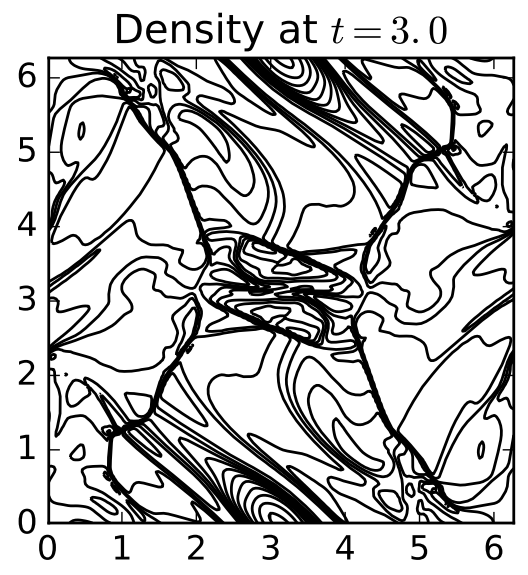

(c)

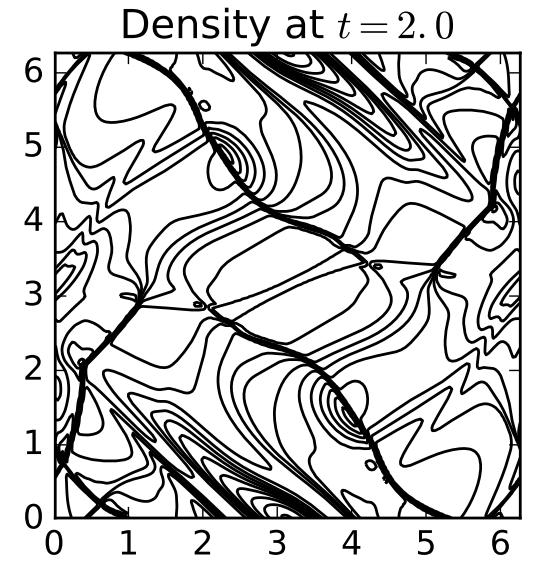

(b)

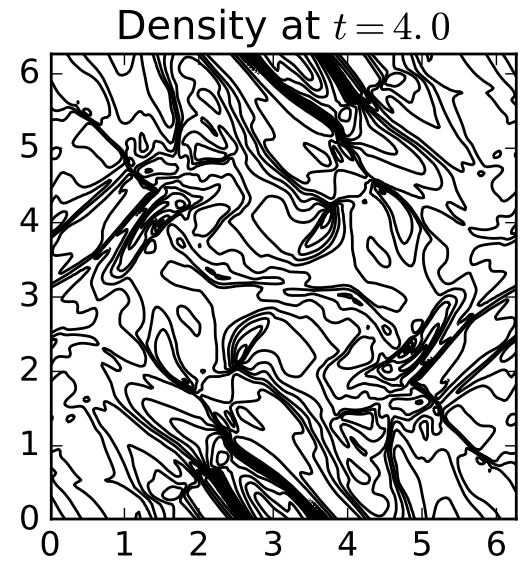

(d)

Figure 4: The Orszag-Tang problem. We show density contour plots at (a) $t=0.5$, (b) $t=2$, (c) $t=3$, (d) $t=4$. The solution is computed using PIF-WENO with constrained transport on and positivity-preserving limiter on, on a $192 \times 192$ mesh. A total of 15 equally spaced contours are used for each graph. 
Control of divergence error of the magnetic field is critical for this test problem. If we turn off the constrained transport, the simulation crashes at $t=1.67$. In Figure 5 we present plots of the density at time $t=1.5$ obtained with different configurations of the numerical schemes. Note the development of the nonphysical features in the solution obtained without constrained transport.

We also note that in this problem, where the positivity-preserving limiter is not needed for the simulation, the limiter leads to little difference in the solution. We present in Figures 5 and 6 plots that demonstrate this. The plots of the type in Figures 4 and 6 are presented in many sources. Our plots agree with results from the literature [8, 57, 34, 35, 58,

\section{4. $2 D$ rotor problem}

This is a two-dimensional test problem that involves low pressure values [28. The setup we use here is the same as the very low $\beta$ version found in 59 . These initial conditions are

$$
\begin{aligned}
& \rho= \begin{cases}10 & \text { if } r \leq 0.1, \\
1+9 \tilde{f}(r) & \text { if } r \in(0.1,0.115), \\
1 & \text { if } r \geq 0.115,\end{cases} \\
& u^{x}= \begin{cases}-10 y+5 & \text { if } r \leq 0.1, \\
(-10 y+5) \tilde{f}(r) & \text { if } r \in(0.1,0.115), \\
0 & \text { if } r \geq 0.115,\end{cases} \\
& u^{y}= \begin{cases}10 x-5 & \text { if } r \leq 0.1 \\
(10 x-5) \tilde{f}(r) & \text { if } r \in(0.1,0.115) \\
0 & \text { if } r \geq 0.115\end{cases} \\
& u^{z}=0, \quad B^{x}=\frac{2.5}{\sqrt{4 \pi}}, \quad B^{y}=0, \quad B^{z}=0, \quad p=10^{-8}, \quad A^{z}=\frac{2.5}{\sqrt{4 \pi}} y,
\end{aligned}
$$

where

$$
r=\sqrt{(x-0.5)^{2}+(y-0.5)^{2}}, \quad \tilde{f}(r)=\frac{1}{3}(23-200 r) .
$$

The computation domain we use is $[0,1] \times[0,1]$, with zeroth order extrapolation on the conserved quantities and first order extrapolation on the magnetic potential as the boundary conditions on all four sides (i.e., conserved quantities at the ghost points are set equal to the last interior point, and values for the magnetic potential are defined through repeated extrapolation of two point stencils).

We compute the solution to a final time of $t=0.27$ using a $400 \times 400$ mesh and present the plots of the magnetic pressure and the magnetic field line in Figure 7 . The magnetic field line plot presented here is the contour plot of $A^{z}$. A total of 50 levels are used in this contour plot. Our result is consistent with the one presented in [59]. We note that the positivity-preserving limiter is necessary to complete this test problem, because otherwise the code fails.

\subsection{Cloud-shock interaction}

The cloud-shock interaction problem is a standard test problem for MHD [8, 57, 32, 33, 34]. The initial conditions are

$$
\begin{aligned}
& \left(\rho, u^{x}, u^{y}, u^{z}, p, B^{x}, B^{y}, B^{z}\right) \\
& \quad= \begin{cases}(3.86859,11.2536,0,0,167.345,0,2.1826182,-2.1826182) & \text { if } x<0.05, \\
(10,0,0,0,1,0,0.56418958,0.56418958) & \text { if } x>0.05 \text { and } r<0.15 \\
(1,0,0,0,1,0,0.56418958,0.56418958) & \text { otherwise }\end{cases}
\end{aligned}
$$

where $r=\sqrt{(x-0.25)^{2}+(y-0.5)^{2}}$ in $2 \mathrm{D}$, and $r=\sqrt{(x-0.25)^{2}+(y-0.5)^{2}+(z-0.5)^{2}}$ in 3D denotes the distance to the center of the stationary cloud. For both problems, we use the initial 


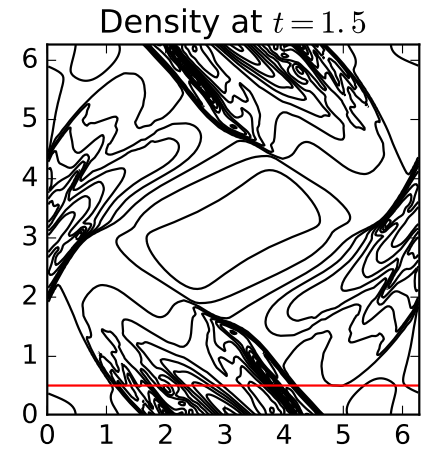

(a)

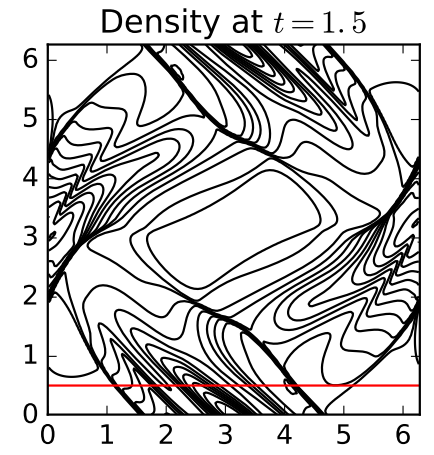

(b)

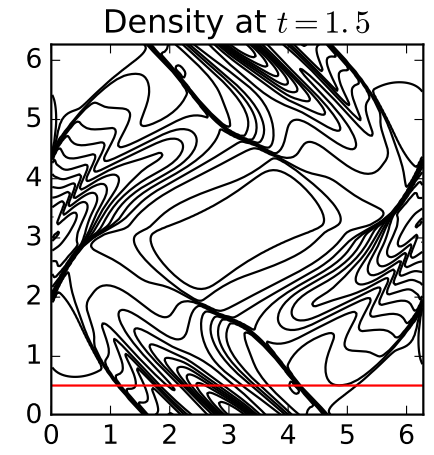

(c)

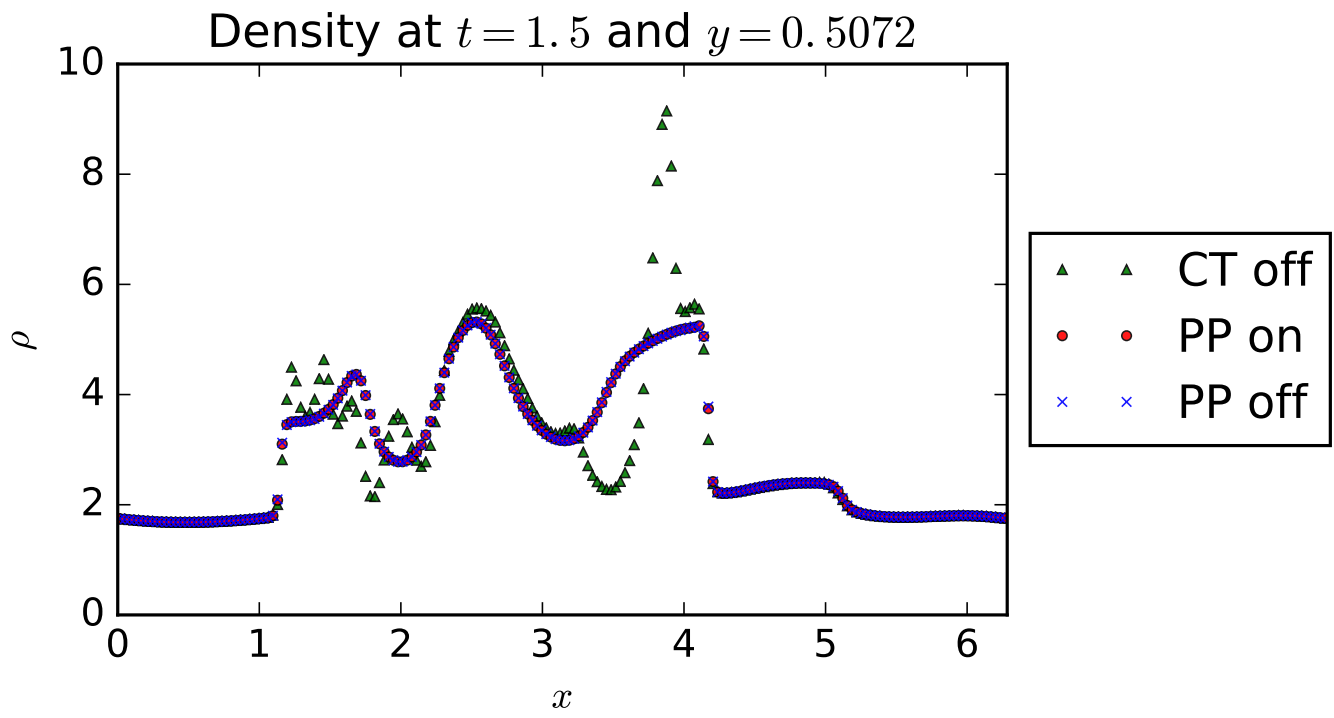

(d)

Figure 5: The Orszag-Tang problem. Density plots at $t=1.5$ for solutions computed using different configurations in the numerical scheme. (a) PIF-WENO, with constrained transport turned off; (b) PIF-WENO with constrained transport and positivity preserving limiter turned on; (c) PIF-WENO with constrained transport turned on and positivity preserving limiter turned off; (d) The slice along $y=0.5072$. The solutions are computed with a $192 \times 192$ mesh. A total of 15 equally spaced contours are used for each of (a), (b), and (c). 


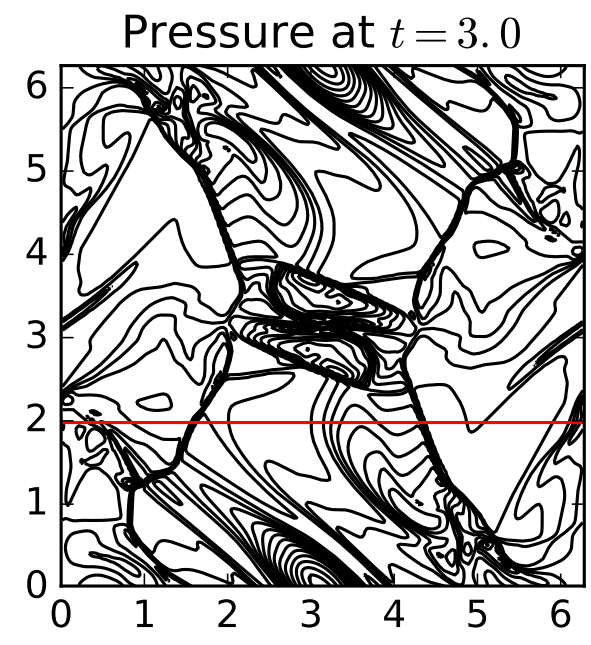

(a)

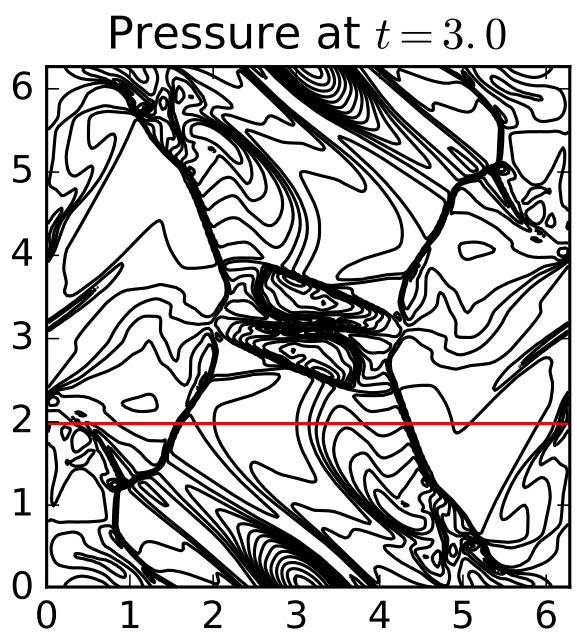

(b)

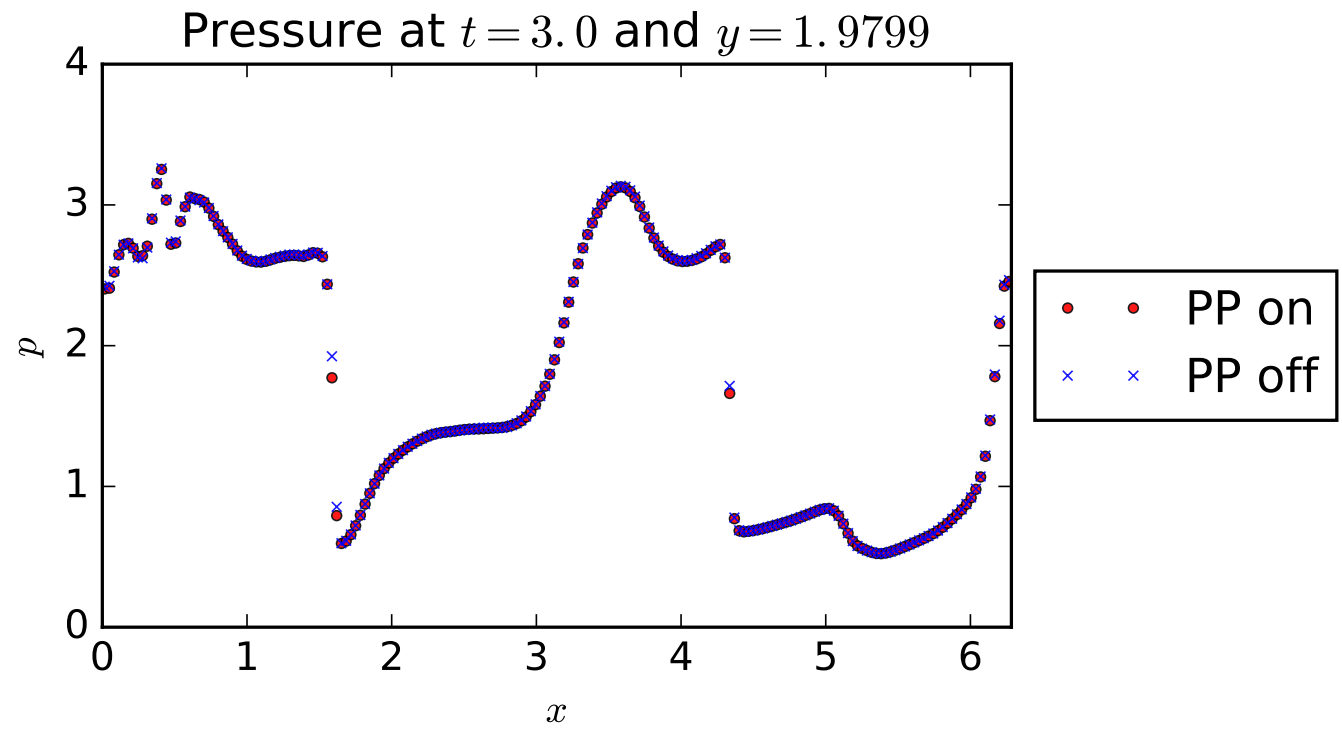

(c)

Figure 6: The Orszag-Tang problem. Pressure plots at $t=3.0$ for solutions computed using different configurations in the numerical scheme. (a) PIF-WENO with constrained transport turned on and positivity preserving limiter turned off; (b) PIF-WENO with constrained transport and positivity preserving limiter turned on; (c) The slice along $y=1.9799$. The solutions are computed with a $192 \times 192$ mesh. A total of 15 equally spaced contours are used for each of (a) and (b). 


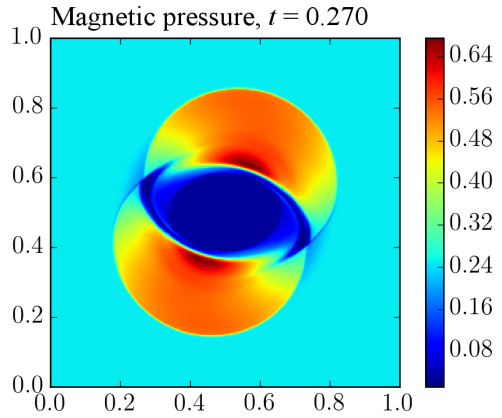

(a)

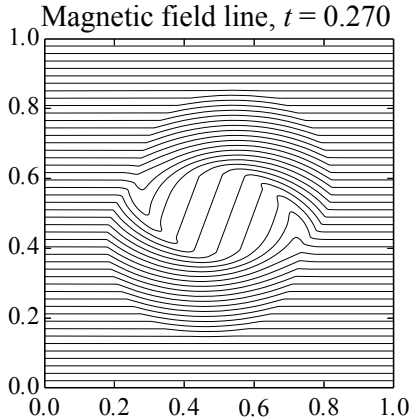

(b)

Figure 7: The 2D rotor problem. Shown here are (a) the pseudocolor plot of the magnetic pressure and (b) the magnetic field line. This solution is computed on a mesh of size $400 \times 400$. Constrained transport and positivitypreserving limiters are turned on.

magnetic potential

$$
A^{x}=0, \quad A^{y}=0, \quad A^{z}= \begin{cases}-2.1826182 x+0.080921431 & \text { if } x \leq 0.05 \\ -0.56418958 x & \text { if } x \geq 0.05\end{cases}
$$

In the 2D case, we only keep track of $A^{z}$.

\subsubsection{Cloud-shock interaction: The 2D problem}

The computational domain we use is $[0,1] \times[0,1]$, with zeroth order extrapolation on the conserved quantities and first order extrapolation on the magnetic potential as the boundary conditions on all four sides (i.e., conserved quantities at the ghost points are set equal to the last interior point, and values for the magnetic potential are defined through repeated extrapolation of two point stencils).

We compute the solution at $t=0.06$ using a $256 \times 256$ mesh. The Schlieren plots of $\ln \rho$ and of $|\mathbf{B}|$ are presented in Figure 8. We note here that the current scheme is able to capture the shock-wave-like structure near $x=0.75$. This is consistent with our previous result in $[8$, and an improvement over earlier results in [57, 34. We also note that the positivity-preserving limiter is not required for this simulation. Nonetheless, we present the result here to demonstrate the high resolution of our method, even when the limiter is turned on.

\subsubsection{Cloud-shock interaction: The 3D problem}

The computational domain for this problem is $[0,1] \times[0,1] \times[0,1]$, with zeroth order extrapolation on the conserved quantities and first order extrapolation on the magnetic potential as the boundary conditions on all six faces (i.e., conserved quantities at the ghost points are set equal to the last interior point, and values for the magnetic potential are defined through repeated extrapolation of two point stencils).

We compute the solution to a final time of time $t=0.06$ on a $256 \times 256 \times 256$ mesh. In Figure 9 , we show the evolution of the density of the solution. We have two remarks on this result. The first is that the shock-wave-like structure near $x=0.75$ at the final time is also visible when we use a $128 \times 128 \times 128$ mesh. The second is that the positivity-preserving limiter is required to run this simulation with $256 \times 256 \times 256$ mesh. The reason is that extra structure that contains very low pressure shows up in this mesh at time $t=0.0378$. This extra structure cannot be observed on the coarser mesh with the method proposed in the current work, nor do we observe it with our previous SSP-RK solver [8]. Therefore, it does not cause trouble for simulations using a $128 \times 128 \times 128$ mesh. 


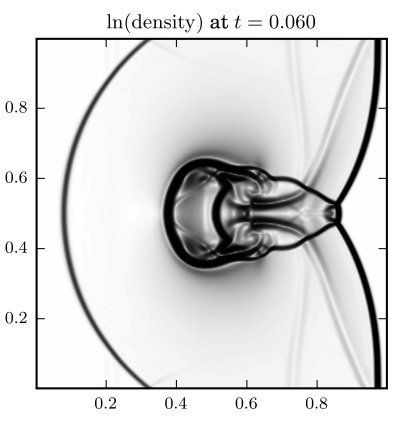

(a)

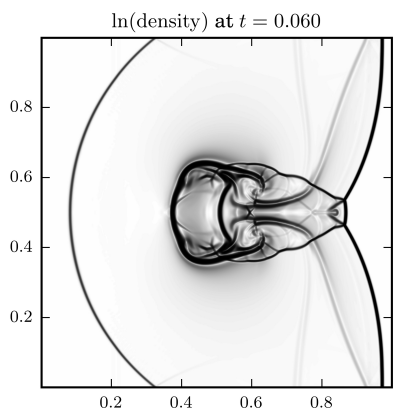

(d)

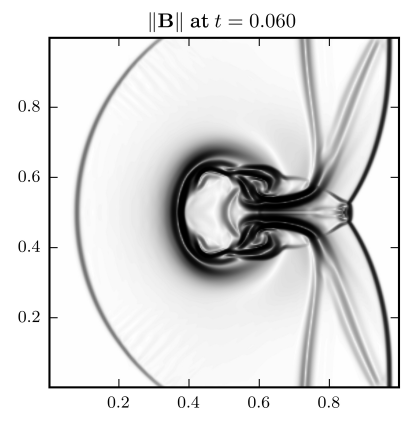

(b)

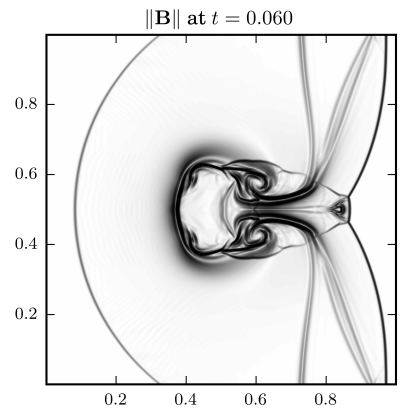

(e)

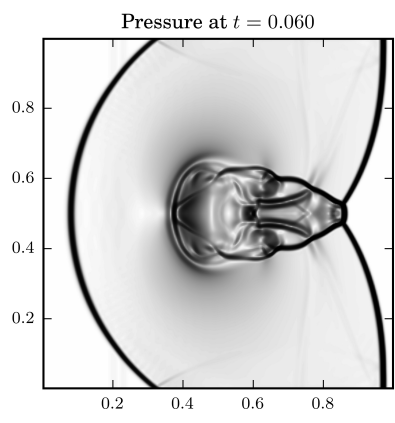

(c)

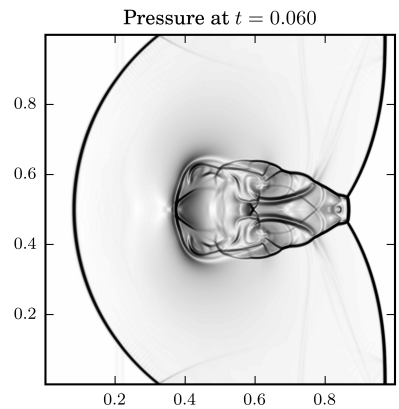

(f)

Figure 8: The 2D cloud-shock interaction problem. Here, we run the solver to a final time of $t=0.06$. In the first three panels, we show Schlieren plots for (a) the natural log of the density, (b) the norm of the magnetic field, and (c) the pressure for a mesh of size $256 \times 256$. The same results for a mesh of size $512 \times 512$ are presented in panels (d)-(f), where we observe much higher resolution for the problem. Constrained transport and positivity-preserving limiter are turned on for both simulations. 

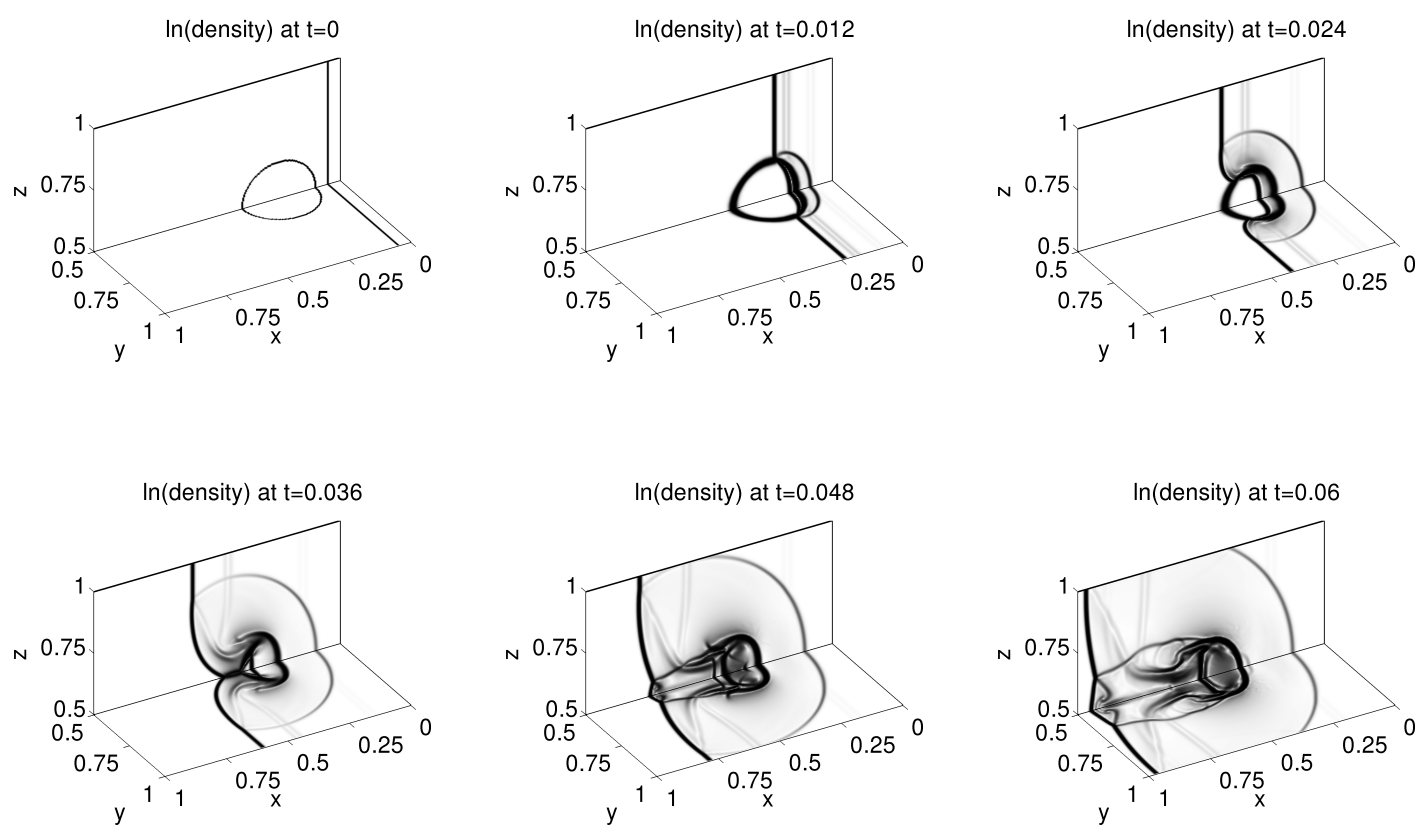

Figure 9: The 3D cloud-shock interaction problem. Schlieren plots of $\ln (\rho)$. The solution here is computed using a $256 \times 256 \times 256$ mesh. Cross-sections at $y=0.5$ and $z=0.5$ for the region $y \geq 0.5$ and $z \geq 0.5$ are shown. Constrained transport and positivity-preserving limiter are turned on.

\subsection{Blast wave example}

In the blast wave problems, strong shocks interact with a low- $\beta$ background, which can cause negative pressure if not handled properly. These problems are often used to test the positivitypreserving capabilities of numerical methods for MHD [14, 28, 42, 60, 61, 62, 63. The initial conditions contain a piecewise defined pressure:

$$
p= \begin{cases}0.1 & r<0.1 \\ 1000 & \text { otherwise }\end{cases}
$$

where $r$ is the distance to the origin, and a constant density, velocity and magnetic field:

$$
\left(\rho, u^{x}, u^{y}, u^{z}, B^{x}, B^{y}, B^{z}\right)=(1,0,0,0,100 / \sqrt{4 \pi} / \sqrt{2}, 100 / \sqrt{4 \pi} / \sqrt{2}, 0) .
$$

The initial magnetic potential is simply

$$
\mathbf{A}=(0,0,100 y / \sqrt{4 \pi} / \sqrt{2}-100 x / \sqrt{4 \pi} / \sqrt{2}) .
$$

In $2 \mathrm{D}$ we only keep track of $A^{z}$, as we do with all the $2 \mathrm{D}$ examples.

\subsubsection{Blast wave example: The 2D problem}

In this section, we present our result on the $2 \mathrm{D}$ version of the blast wave problem. The computational domain is $[-0.5,0.5] \times[-0.5,0.5]$, with zeroth order extrapolation on the conserved quantities and first order extrapolation on the magnetic potential as the boundary conditions on all four sides (i.e., conserved quantities at the ghost points are set equal to the last interior point, and values for the magnetic potential are defined through repeated extrapolation of two point stencils). 


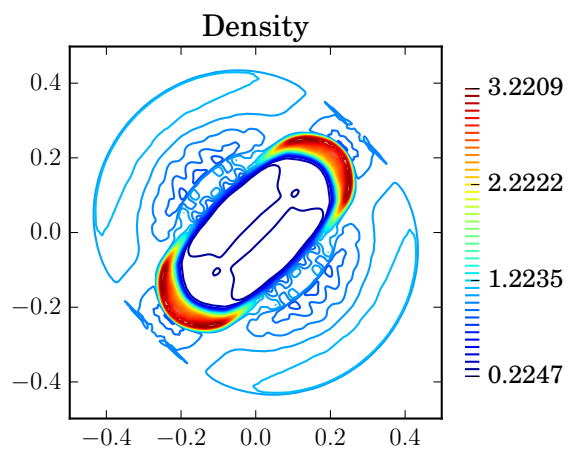

(a)

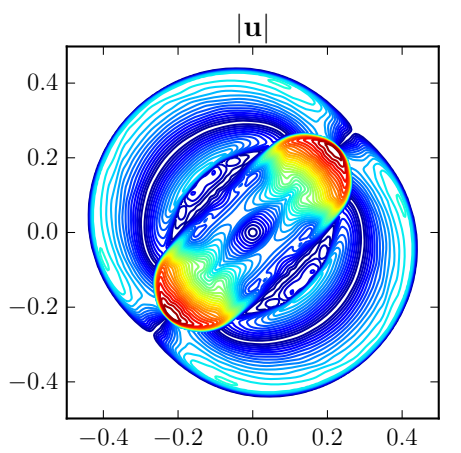

(c)

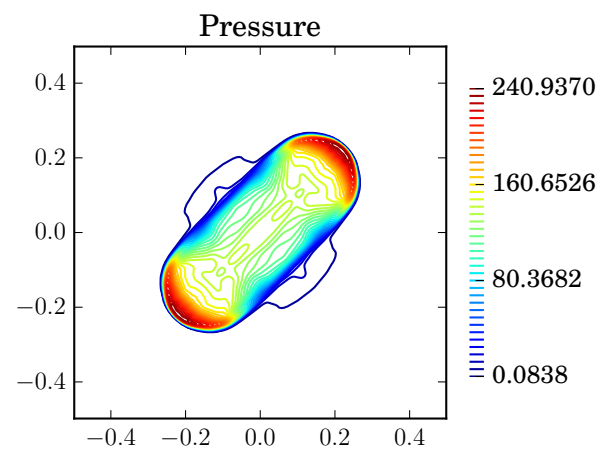

(b)

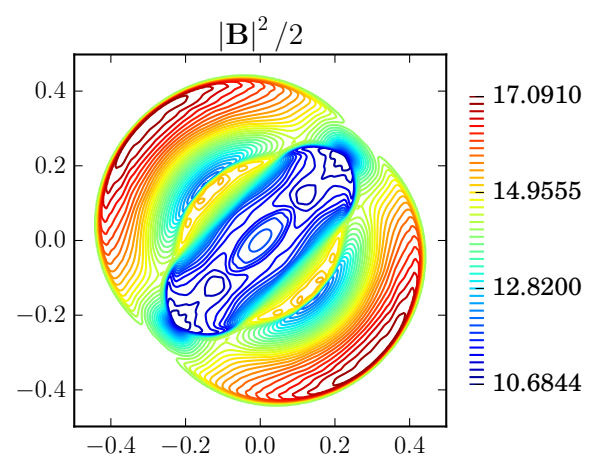

(d)

Figure 10: 2D blast problem. Shown here are the contour plots at $t=0.01$ of (a) density, (b) thermal pressure, (c) magnitude of velocity, and (d) magnetic pressure. A total of 40 equally spaced contours ranging from the min to the max of the function are used for each plot. The mesh size is $256 \times 256$. 
Density at $\mathrm{t}=0.01$

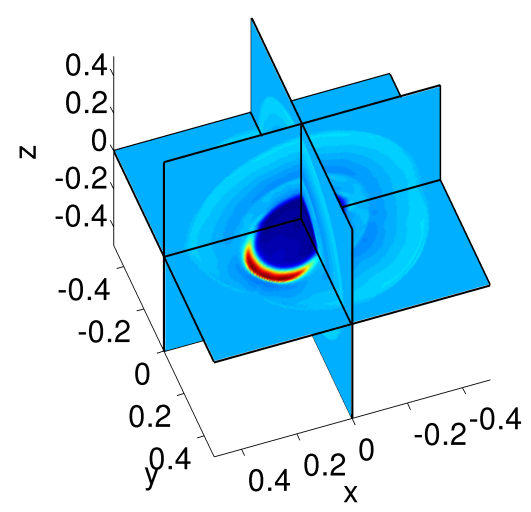

(a)
Pressure at $\mathrm{t}=0.01$

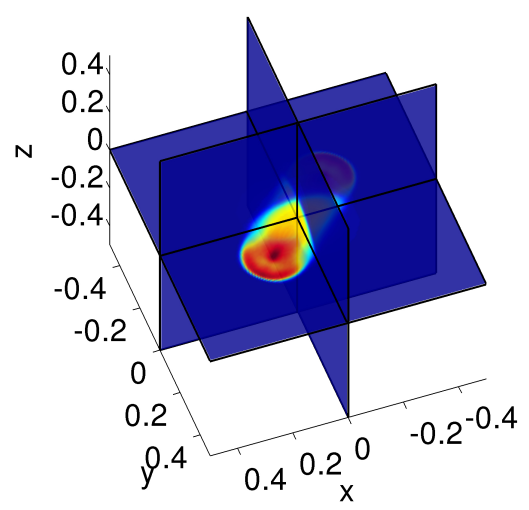

(b)

Figure 11: 3D blast problem. Shown here are the pseudocolor plots at $t=0.01$ of (a) density and (b) pressure. The mesh size is $150 \times 150 \times 150$. In Figure 12 we plot a cut of the solution along $z=0$. The positivity-preserving limiter is required to simulate this problem.

Results for the solution computed to a final time of $t=0.01$ on a $256 \times 256$ mesh are presented in Figure 10. There, we display contour plots of $\rho, p,|\mathbf{u}|$, and $|\mathbf{B}|$. These plots are comparable to the previous results in 42 . We note that negative pressure occurs right in the first step if positivity-preserving limiter is turned off.

\subsubsection{Blast wave example: The $3 D$ problem}

For the $3 \mathrm{D}$ version of the blast wave problem, we choose the computational domain to be $[-0.5,0.5] \times[-0.5,0.5] \times[-0.5,0.5]$ with zeroth order extrapolation on the conserved quantities and first order extrapolation on the magnetic potential as the boundary conditions on all six faces (i.e., conserved quantities at the ghost points are set equal to the last interior point, and values for the magnetic potential are defined through repeated extrapolation of two point stencils).

The solution at $t=0.01$ is computed using a $150 \times 150 \times 150$ mesh. We present in Figure 11 the plots of the density and pressure, and also in Figure 12 the contour plots of the slice at $z=0$ of the density, pressure, velocity, and magnetic pressure. These results are comparable to those found in $42,60,62,63$. We note here that negative pressure occurs in the second time step if the positivity-preserving limiter is turned off.

\subsection{Errors in energy conservation}

When the positivity-preserving limiter is turned on, we make use of an energy correction step in Eqn. 13 in order to keep the pressure the same as before the magnetic field correction. This breaks the conservation of the energy, and therefore we investigate the effect of this step for several test problems. Because (global) energy conservation only holds for problems that have either periodic boundary conditions or constant values near the boundary throughout the entire simulation, we only choose problems with this property for our test cases.

For the $2 \mathrm{D}$ problems, the (relative) energy conservation error at time $t=t^{n}$ is defined as

$$
\text { Energy conservation error }:=\frac{\left|\sum_{i, j}\left(\mathcal{E}_{i, j}^{n}-\mathcal{E}_{i, j}^{0}\right)\right|}{\sum_{i, j} \mathcal{E}_{i, j}^{0}},
$$

and the energy conservation errors for the 3D problems are defined similarly. 


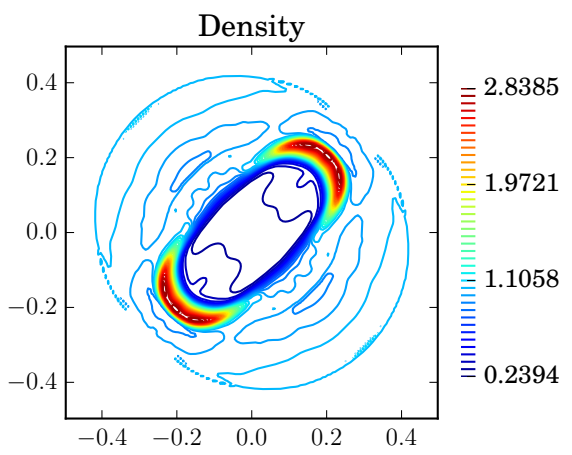

(a)

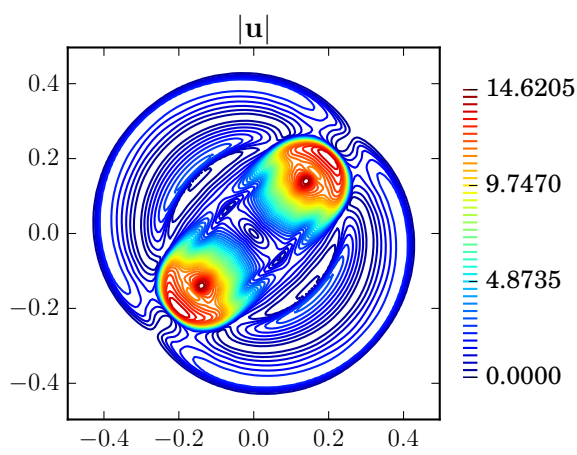

(c)

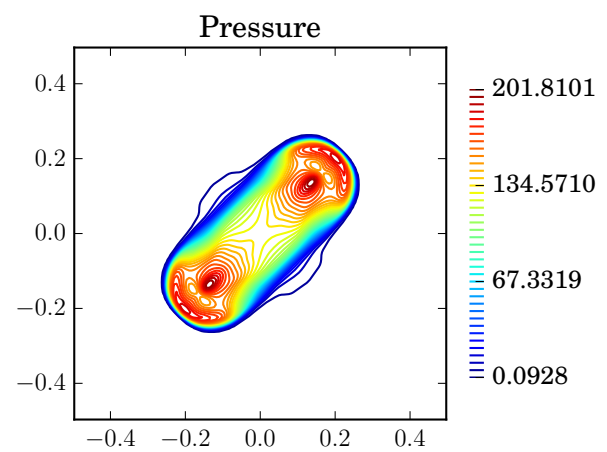

(b)

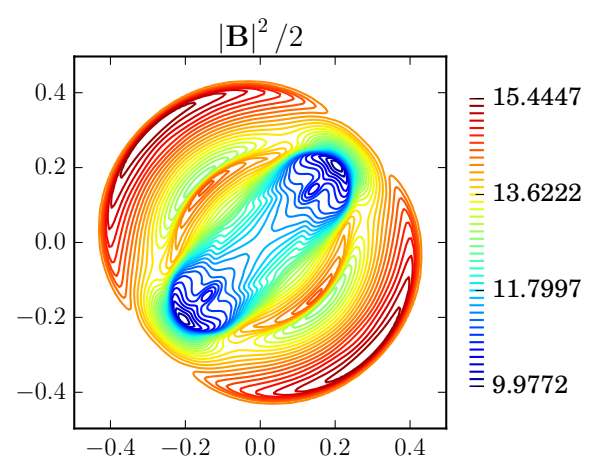

(d)

Figure 12: 3D blast problem. Shown are the contour plots at time $t=0.01$ cut at $z=0$ of (a) density, (b) thermal pressure, (c) norm of velocity, and (d) magnetic pressure. The solution is obtained using a $150 \times 150 \times 150$ mesh. A total of 40 equally spaced contours are used for each plot. 


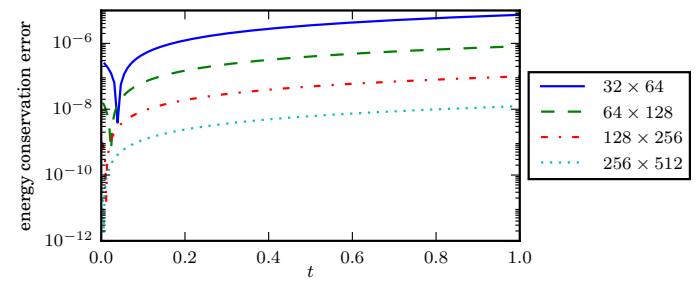

(a)

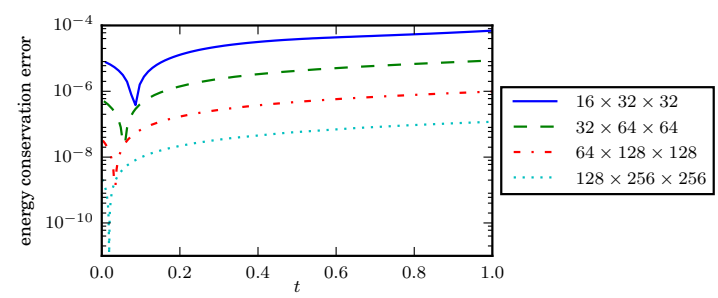

(b)

Figure 13: Energy conservation errors for the smooth Alfvén test cases. Shown here are results for the (a) 2D smooth Alfvén, and (b) 3D smooth Alfvén test cases. In order to extract the errors, we plot the results on a semi-log scale because otherwise the results are indiscernible from the $t$-axis. For this smooth test case, the effect of the the energy correction step (and hence the positivity-preserving limiter) is negligible because the solution remains smooth for the entire simulation.

Results for the 2D and 3D smooth Alfvén test case are presented in Figure 13 , where we observe negligible errors produced by the energy correction step. We attribute this to the fact that this problem retains a smooth solution for the entirety of the simulation. Results for problems with shocks and vortices are presented in Figure 14. where we find non-zero errors. For each of these test problems, we present the results from several different sizes of meshes. All the problems are run to the final time found in Sections 6.1 6.6 save one. For the 2D Orszag-Tang problem, we run the simulations to a much later time of $t=30$ in order to quantify the energy conservation errors for a long time simulation on a non-trivial problem.

Finally, in Figure 15 we also include the conservation errors when the positivity-preserving limiter is turned off. We observe that the solver retains total energy up to machine roundoff errors, as should be the case.

We note the following patterns in the energy conservation errors:

- The errors are below $1 \%$ for all the test problems in the duration of the simulations presented;

- When the positivity-preserving limiter is turned on, the errors grow linearly in time and decrease as the mesh is refined.

We therefore conclude that the violation in energy conservation introduced by the positivity-preserving limiter is insignificant for the problems tested in this work.

\section{Conclusion and future work}

In this paper we propose a high-order single-stage single-step positivity-preserving method for the ideal magnetohydrodynamic equations. The base scheme uses a finite difference WENO method with a Lax-Wendroff time discretization that is based on the Picard integral formulation of hyperbolic conservation laws. A discrete divergence-free condition on the magnetic field is found by using an unstaggered constrained transport method that evolves a vector potential alongside the conserved quantities on the same mesh as the conserved variables. This vector potential is evolved with a modified version of a finite difference Lax-Wendroff WENO method that was originally developed for Hamilton-Jacobi equations. This allows us to define non-oscillatory derivatives for the magnetic field. To further enhance the robustness of our scheme, a flux limiter is added to preserve the positivity of the density and pressure. Unlike our previous solvers that are based on SSP-RK time stepping [8, 42, this solver does not require the use of intermediate stages. This reduces the total storage required for the method, and may lead to more efficient implementation for an AMR setting. Moreover, we need only apply one WENO reconstruction per time step for the fluid variables, whereas the third and fourth-order solvers in 8 require three and ten, respectively, WENO reconstructions 


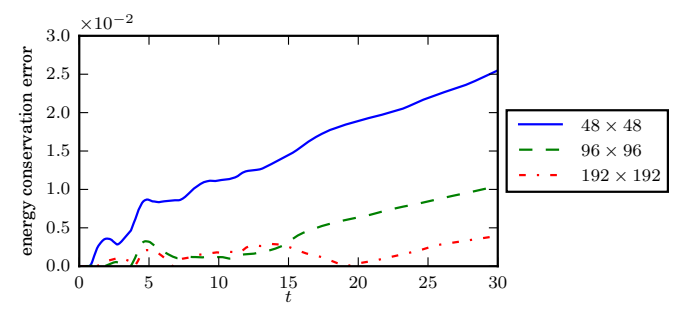

(a)

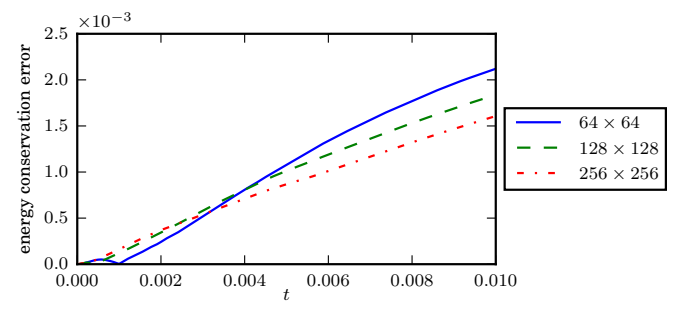

(c)

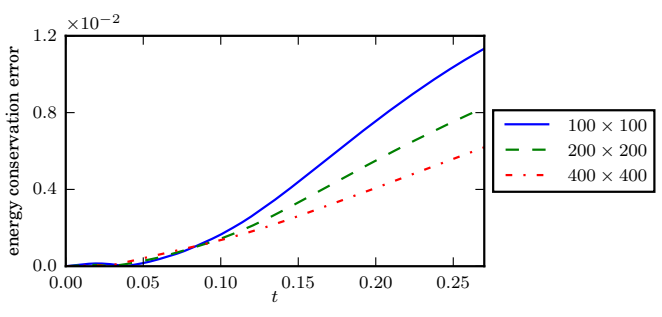

(b)

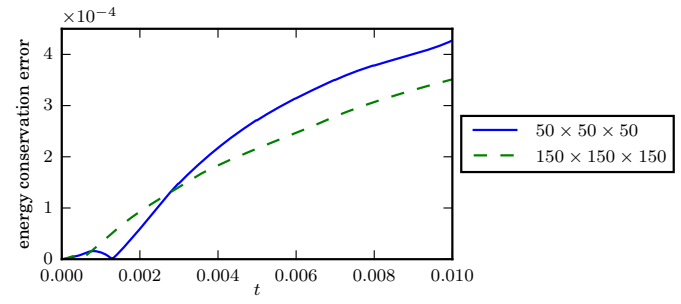

(d)

Figure 14: Energy conservation errors. Shown here are conservation errors when the positivity-preserving limiter (and hence the energy correction step) is turned on. (a) 2D Orszag-Tang problem, (b) 2D rotor problem, (c) 2D blast problem, and (d) 3D blast problem. Note that the rotor and blast problems require the application of a positivitypreserving limiter in order to run, but this comes at the expense of losing energy conservation. For the Orszag-Tang test problem, we run to a late final time. Again, we observe that the errors in energy conservation decrease as the mesh is refined.

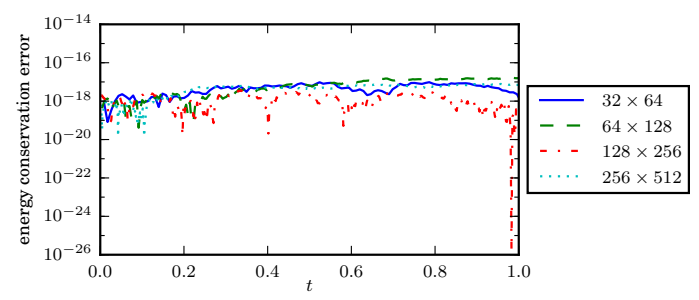

(a)

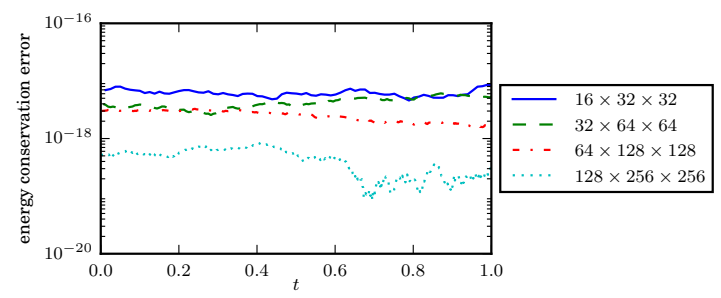

(b)

Figure 15: Energy conservation errors. Here, we show results for conservation errors when the positivity-preserving limiter (and hence the energy correction step) is turned off. The solver analytically conserves the discrete total energy up to machine precision. Shown here are results for the (a) 2D smooth Alfvén, and (b) 3D smooth Alfvén test cases. Note the logarithmic scale for the axes, and that these errors are numerically conserved up to machine precision. 
per time step. Numerical results show that our scheme has the expected high-order accuracy for smooth problems, and is capable of solving some very stringent test problems, even when low density and pressure are present. Future work includes embedding this scheme into an AMR framework, extending the scheme to curvilinear meshes to accommodate complex geometry, and incorporate non-ideal terms in the MHD equations by means of a semi-implicit solver.

\section{Acknowledgements}

We would like to thank the anonymous reviewers for their thoughtful comments and suggestions to improve this work. This work was supported by: AFOSR grants FA9550-15-1-0282, and FA955012-1-0343; the Michigan State University Foundation; NSF grant DMS-1418804; and Oak Ridge National Laboratory (ORAU HPC LDRD).

\section{References}

[1] D. D. Schnack. Lectures in magnetohydrodynamics, volume 780 of Lecture Notes in Physics. Springer-Verlag, Berlin, 2009. With an appendix on extended MHD.

[2] P. Londrillo and L. Del Zanna. High-order upwind schemes for multidimensional magnetohydrodynamics. The Astrophysical Journal, 530(1):508, 2000.

[3] Guang-Shan Jiang and Cheng-chin Wu. A high-order WENO finite difference scheme for the equations of ideal magnetohydrodynamics. J. Comput. Phys., 150(2):561-594, 1999.

[4] Shengtai Li and Hui Li. A modern code for solving magnetohydrodynamics or hydrodynamic equations. Technical report, Los Alamos National Lab., 2003.

[5] Dinshaw S Balsara. Divergence-free reconstruction of magnetic fields and WENO schemes for magnetohydrodynamics. Journal of Computational Physics, 228(14):5040-5056, 2009.

[6] Yiqing Shen, Gecheng Zha, and Manuel A Huerta. E-CUSP scheme for the equations of ideal magnetohydrodynamics with high order WENO scheme. Journal of Computational Physics, 231(19):6233-6247, 2012.

[7] Dinshaw S. Balsara, Chad Meyer, Michael Dumbser, Huijing Du, and Zhiliang Xu. Efficient implementation of ADER schemes for Euler and magnetohydrodynamical flows on structured meshes-speed comparisons with Runge-Kutta methods. J. Comput. Phys., 235:934-969, 2013.

[8] Andrew J. Christlieb, James A. Rossmanith, and Qi Tang. Finite difference weighted essentially non-oscillatory schemes with constrained transport for ideal magnetohydrodynamics. $J$. Comput. Phys., 268:302-325, 2014.

[9] Yuxi Chen, Gábor Tóth, and Tamas I. Gombosi. A fifth-order finite difference scheme for hyperbolic equations on block-adaptive curvilinear grids. J. Comput. Phys., 305:604-621, 2016.

[10] Peter McCorquodale and Phillip Colella. A high-order finite-volume method for conservation laws on locally refined grids. Commun. Appl. Math. Comput. Sci., 6(1):1-25, 2011.

[11] Chaopeng Shen, Jing-Mei Qiu, and Andrew Christlieb. Adaptive mesh refinement based on high order finite difference WENO scheme for multi-scale simulations. J. Comput. Phys., 230(10):3780-3802, 2011.

[12] Cheng Wang, XinZhuang Dong, and Chi-Wang Shu. Parallel adaptive mesh refinement method based on WENO finite difference scheme for the simulation of multi-dimensional detonation. $J$. Comput. Phys., 298:161-175, 2015. 
[13] Phillip Colella. Multidimensional upwind methods for hyperbolic conservation laws. J. Comput. Phys., 87(1):171-200, 1990.

[14] Dinshaw S. Balsara, Tobias Rumpf, Michael Dumbser, and Claus-Dieter Munz. Efficient, high accuracy ADER-WENO schemes for hydrodynamics and divergence-free magnetohydrodynamics. J. Comput. Phys., 228(7):2480-2516, 2009.

[15] Peter Lax and Burton Wendroff. Systems of conservation laws. Comm. Pure Appl. Math., 13:217-237, 1960.

[16] V. A. Titarev and E. F. Toro. ADER: arbitrary high order Godunov approach. J. Sci. Comput., 17(1-4):609-618, 2002.

[17] E. F. Toro, R. C. Millington, and L. A. M. Nejad. Towards very high order Godunov schemes. In Godunov methods (Oxford, 1999), pages 907-940. Kluwer/Plenum, New York, 2001.

[18] Jianxian Qiu and Chi-Wang Shu. Finite difference WENO schemes with Lax-Wendroff-type time discretizations. SIAM J. Sci. Comput., 24(6):2185-2198, 2003.

[19] Jianxian Qiu, Michael Dumbser, and Chi-Wang Shu. The discontinuous Galerkin method with Lax-Wendroff type time discretizations. Comput. Methods Appl. Mech. Engrg., 194(4244):4528-4543, 2005.

[20] William D Henshaw. A high-order accurate parallel solver for Maxwell's equations on overlapping grids. SIAM Journal on Scientific Computing, 28(5):1730-1765, 2006.

[21] Jeffrey W Banks and William D Henshaw. Upwind schemes for the wave equation in secondorder form. Journal of Computational Physics, 231(17):5854-5889, 2012.

[22] W. Boscheri, M. Dumbser, and D. S. Balsara. High-order ADER-WENO ALE schemes on unstructured triangular meshes - application of several node solvers to hydrodynamics and magnetohydrodynamics. Internat. J. Numer. Methods Fluids, 76(10):737-778, 2014.

[23] Andrew J. Christlieb, Yaman Güçlü, and David C. Seal. The Picard integral formulation of weighted essentially nonoscillatory schemes. SIAM J. Numer. Anal., 53(4):1833-1856, 2015.

[24] J. U. Brackbill and D. C. Barnes. The effect of nonzero $\nabla \cdot \mathbf{B}$ on the numerical solution of the magnetohydrodynamic equations. J. Comput. Phys., 35(3):426-430, 1980.

[25] Tamas I Gombosi, Kenneth G Powell, and Darren L De Zeeuw. Axisymmetric modeling of cometary mass loading on an adaptively refined grid: Mhd results. J. Geophys. Res. - Space, 99(A11):21525-21539, 1994.

[26] A. Dedner, F. Kemm, D. Kröner, C.-D. Munz, T. Schnitzer, and M. Wesenberg. Hyperbolic Divergence Cleaning for the MHD Equations. Journal of Computational Physics, 175(2):645673, January 2002.

[27] Dinshaw S Balsara. Second-order-accurate schemes for magnetohydrodynamics with divergencefree reconstruction. Astrophys. J. Suppl. S., 151(1):149, 2004.

[28] Dinshaw S Balsara and Daniel S Spicer. A staggered mesh algorithm using high order Godunov fluxes to ensure solenoidal magnetic fields in magnetohydrodynamic simulations. J. Comput. Phys., 149(2):270-292, 1999.

[29] Wenlong Dai and Paul R Woodward. On the divergence-free condition and conservation laws in numerical simulations for supersonic magnetohydrodynamical flows. Astrophys. J., 494(1):317, 1998. 
[30] Charles R Evans and John F Hawley. Simulation of magnetohydrodynamic flows - a constrained transport method. Astrophys. J., 332:659-677, 1988.

[31] Michael Fey and Manuel Torrilhon. A constrained transport upwind scheme for divergence-free advection. In Hyperbolic problems: theory, numerics, applications, pages 529-538. Springer, Berlin, 2003.

[32] Christiane Helzel, James A. Rossmanith, and Bertram Taetz. An unstaggered constrained transport method for the 3D ideal magnetohydrodynamic equations. J. Comput. Phys., 230(10):3803$3829,2011$.

[33] Christiane Helzel, James A. Rossmanith, and Bertram Taetz. A high-order unstaggered constrained-transport method for the three-dimensional ideal magnetohydrodynamic equations based on the method of lines. SIAM J. Sci. Comput., 35(2):A623-A651, 2013.

[34] James A. Rossmanith. An unstaggered, high-resolution constrained transport method for magnetohydrodynamic flows. SIAM J. Sci. Comput., 28(5):1766-1797, 2006.

[35] Gábor Tóth. The $\nabla \cdot B=0$ constraint in shock-capturing magnetohydrodynamics codes. $J$. Comput. Phys., 161(2):605-652, 2000.

[36] S. C. Jardin. Review of implicit methods for the magnetohydrodynamic description of magnetically confined plasmas. J. Comput. Phys., 231(3):822-838, 2012.

[37] Jianxian Qiu. WENO schemes with Lax-Wendroff type time discretizations for Hamilton-Jacobi equations. J. Comput. Appl. Math., 200(2):591-605, 2007.

[38] Dinshaw S. Balsara and Daniel Spicer. Maintaining pressure positivity in magnetohydrodynamic simulations. J. Comput. Phys., 148(1):133-148, 1999.

[39] P. Janhunen. A positive conservative method for magnetohydrodynamics based on HLL and Roe methods. J. Comput. Phys., 160(2):649-661, 2000.

[40] Dinshaw S. Balsara. Self-adjusting, positivity preserving high order schemes for hydrodynamics and magnetohydrodynamics. J. Comput. Phys., 231(22):7504-7517, 2012.

[41] Yue Cheng, Fengyan Li, Jianxian Qiu, and Liwei Xu. Positivity-preserving DG and central DG methods for ideal MHD equations. J. Comput. Phys., 238:255-280, 2013.

[42] Andrew J. Christlieb, Yuan Liu, Qi Tang, and Zhengfu Xu. Positivity-preserving finite difference weighted ENO schemes with constrained transport for ideal magnetohydrodynamic equations. SIAM J. Sci. Comput., 37(4):A1825-A1845, 2015.

[43] Zhengfu Xu. Parametrized maximum principle preserving flux limiters for high order schemes solving hyperbolic conservation laws: one-dimensional scalar problem. Math. Comp., 83(289):2213-2238, 2014.

[44] Jay P. Boris and David L. Book. Flux-corrected transport. I. SHASTA, a fluid transport algorithm that works [J. Comput. Phys. 11 (1973), no. 1, 38-69]. J. Comput. Phys., 135(2):170186, 1997. With an introduction by Steven T. Zalesak, Commemoration of the 30th anniversary \{of J. Comput. Phys.\}.

[45] Steven T. Zalesak. Fully multidimensional flux-corrected transport algorithms for fluids. $J$. Comput. Phys., 31(3):335-362, 1979.

[46] Xiangxiong Zhang and Chi-Wang Shu. On positivity-preserving high order discontinuous Galerkin schemes for compressible Euler equations on rectangular meshes. J. Comput. Phys., 229(23):8918-8934, 2010. 
[47] Andrew J. Christlieb, Yuan Liu, Qi Tang, and Zhengfu Xu. High order parametrized maximumprinciple-preserving and positivity-preserving WENO schemes on unstructured meshes. J. Comput. Phys., 281:334-351, 2015.

[48] David C. Seal, Qi Tang, Zhengfu Xu, and Andrew J. Christlieb. An explicit high-order singlestage single-step positivity-preserving finite difference WENO method for the compressible Euler equations. Journal of Scientific Computing, pages 1-20, 2015.

[49] M. Brio and C. C. Wu. Characteristic fields for the equations of magnetohydrodynamics. In Nonstrictly hyperbolic conservation laws (Anaheim, Calif., 1985), volume 60 of Contemp. Math., pages 19-23. Amer. Math. Soc., Providence, RI, 1987.

[50] Kenneth G. Powell, Philip L. Roe, Timur J. Linde, Tamas I. Gombosi, and Darren L. De Zeeuw. A solution-adaptive upwind scheme for ideal magnetohydrodynamics. J. Comput. Phys., 154(2):284-309, 1999.

[51] Constantine M. Dafermos. Hyperbolic conservation laws in continuum physics, volume 325 of Grundlehren der Mathematischen Wissenschaften [Fundamental Principles of Mathematical Sciences]. Springer-Verlag, Berlin, third edition, 2010.

[52] Stanley Osher and Chi-Wang Shu. High-order essentially nonoscillatory schemes for HamiltonJacobi equations. SIAM J. Numer. Anal., 28(4):907-922, 1991.

[53] Guang-Shan Jiang and Chi-Wang Shu. Efficient implementation of weighted ENO schemes. $J$. Comput. Phys., 126(1):202-228, 1996.

[54] Chi-Wang Shu. Essentially non-oscillatory and weighted essentially non-oscillatory schemes for hyperbolic conservation laws. In Advanced numerical approximation of nonlinear hyperbolic equations (Cetraro, 1997), volume 1697 of Lecture Notes in Math., pages 325-432. Springer, Berlin, 1998.

[55] Chi-Wang Shu and Stanley Osher. Efficient implementation of essentially non-oscillatory shockcapturing schemes. Journal of Computational Physics, 77(2):439-471, 1988.

[56] Chi-Wang Shu and Stanley Osher. Efficient implementation of essentially non-oscillatory shockcapturing schemes,II. Journal of Computational Physics, 83(1):32-78, July 1989.

[57] Wenlong Dai and Paul R. Woodward. A simple finite difference scheme for multidimensional magnetohydrodynamical equations. J. Comput. Phys., 142(2):331-369, 1998.

[58] Andrew L. Zachary, Andrea Malagoli, and Phillip Colella. A higher-order Godunov method for multidimensional ideal magnetohydrodynamics. SIAM J. Sci. Comput., 15(2):263-284, 1994.

[59] K. Waagan. A positive MUSCL-Hancock scheme for ideal magnetohydrodynamics. J. Comput. Phys., 228(23):8609-8626, 2009.

[60] Thomas A. Gardiner and James M. Stone. An unsplit Godunov method for ideal MHD via constrained transport in three dimensions. J. Comput. Phys., 227(8):4123-4141, 2008.

[61] Fengyan Li, Liwei Xu, and Sergey Yakovlev. Central discontinuous Galerkin methods for ideal MHD equations with the exactly divergence-free magnetic field. J. Comput. Phys., 230(12):4828-4847, 2011.

[62] Andrea Mignone and Petros Tzeferacos. A second-order unsplit Godunov scheme for cellcentered MHD: the CTU-GLM scheme. J. Comput. Phys., 229(6):2117-2138, 2010.

[63] U Ziegler. A central-constrained transport scheme for ideal magnetohydrodynamics. J. Comput. Phys., 196(2):393-416, 2004. 